\title{
Susceptibility of F/A-18 Flight Controllers to the Falling-Leaf Mode: Linear Analysis
}

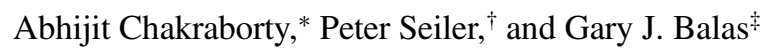 \\ University of Minnesota, Minneapolis, Minnesota 55455
}

DOI: $\underline{10.2514 / 1.50674}$

\begin{abstract}
The F/A-18 Hornet aircraft with the original flight control law exhibited a nonlinear out-of-control phenomenon known as the falling-leaf mode. This unstable mode was suppressed by modifying the control law. This paper employs the falling-leaf phenomenon as an example to investigate the applicability of linear analysis tools for detecting inherently nonlinear phenomenon. A high-fidelity nonlinear model of the F/A-18 is developed for controller analysis using F/A-18 High-Alpha Research Vehicle aerodynamic data in the open literature. A variety of linear analysis methods are used to investigate the robustness properties of the original (baseline) and the revised F/A-18 flight control law at different trim points. Classical analyses, e.g., gain and phase margins, do not indicate a significant improvement in robustness properties of the revised control law over the baseline design. However, advanced robustness analyses, e.g., $\mu$ analysis, indicate that the revised control law is better able to handle the cross-coupling and variations in the dynamics than the baseline design.
\end{abstract}

\section{Nomenclature}

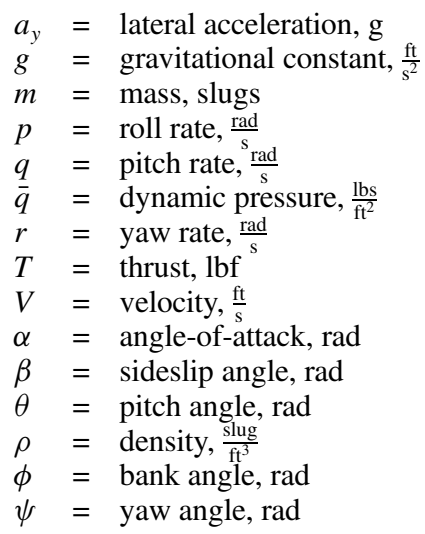

\section{Introduction}

$\mathbf{T}$ HE U.S. Navy F/A-18 A/B/C/D Hornet aircraft with its baseline flight control law experienced a number of out-of-control flight departures since the early 1980 s $[1,2]$. Many of these incidents were associated with a falling-leaf mode instability of the aircraft [2]. The falling-leaf mode in the Hornet went undetected in spite of extensive validation and verification before entry into the fleet. Inability to accurately model the complex aerodynamic characteristics of the aircraft are often attributed for the failure to identify the falling-leaf motion [1,2]. An extensive revision of the original (baseline) flight control law was performed by NAVAIR and Boeing in 2001 to suppress departure phenomenon, improve maneuvering performance and to expand the flight envelope of the vehicle [2]. The revised control law was implemented and successfully flight tested on the F/

Presented as Paper 2009-5675 at the AIAA Guidance, Navigation, and Control Conference, Chicago IL, 10-13 August 2009; received 7 May 2010; revision received 7 September 2010; accepted for publication 7 September 2010. Copyright $@ 2010$ by Abhijit Chakraborty, Peter Seiler, Gary J. Balas. Published by the American Institute of Aeronautics and Astronautics, Inc., with permission. Copies of this paper may be made for personal or internal use, on condition that the copier pay the $\$ 10.00$ per-copy fee to the Copyright Clearance Center, Inc., 222 Rosewood Drive, Danvers, MA 01923; include the code 0731-5090/11 and \$10.00 in correspondence with the CCC.

${ }^{*}$ Graduate Research Assistant, Department of Aerospace Engineering and Mechanics; chakrab@aem.umn.edu.

${ }^{\dagger}$ Senior Research Associate, Department of Aerospace Engineering and Mechanics; seiler@aem.umn.edu.

Frofessor, Department of Aerospace Engineering and Mechanics; balas@aem.umn.edu. Associate Fellow AIAA.
A-18 E/F Super Hornet aircraft. The Super Hornet has similar aerodynamic and inertial characteristics as the Hornet [2] . These flight tests included aggressive maneuvers that demonstrated successful suppression of the falling-leaf motion by the revised control law.

The falling-leaf mode is a nonlinear aerodynamic characteristics of the F/A-18 Hornet which required modification of its flight control law. The nonlinear falling-leaf mode poses a great challenge to understand its interaction with the flight control system. In this paper, the falling-leaf mode is treated as an example to investigate if the standard practices for flight control validation and verification are rich enough to safeguard against nonlinear phenomenon in the safety critical flight systems.

Current practice for flight control validation relies heavily on linear analyses and nonlinear, high-fidelity simulations. The linear analyses amount to assessing the closed-loop stability and performance characteristics of the aircraft flight control system around numerous trim conditions using linear analysis tools. The linear analysis methods include stability margins, robustness analysis and worst-case analysis. The objective of the paper is to understand the ability of linear robustness analysis techniques to identify the suppression of the falling-leaf mode on the F/A-18 by the revised flight controller.

A nonlinear six-degree-of-freedom (DOF) mathematical model of the F/A-18 aircraft is developed in this paper. The aerodynamic model of the aircraft is formulated as polynomial functions of angleof-attack based on data available in the open literature [3-8]. Moreover, the sideslip dependence of the aerodynamic model has been simplified due to lack of availability of sideslip data in the open literature. The nonlinear model presented exhibits nonlinear characteristics of the aircraft including the falling-leaf. However, it has limitations and does not capture all the nonlinear dynamic characteristics of the F/A-18 aircraft. The model used in this paper is able to generate falling-leaf like responses of the aircraft. Hence, it can be used as an example to address the issue if linear analysis is sufficient to uncover nonlinear phenomenon in the flight system.

A comparison of the linear robustness properties of the original (baseline) and the revised flight control law is investigated. Classical linear analyses, e.g., gain and phase margin, do not indicate a significant improvement in robustness properties of the revised control law over the baseline design. Advanced linear robustness analyses, e.g. $\mu$ analyses, indicate that the revised design is better able to handle the cross-coupling and variations in the dynamics than the baseline design. There is a gap in the analysis as the falling-leaf mode is a nonlinear phenomenon and cannot be duplicated with linear aircraft models. This motivates the need for the application of nonlinear analysis tools to compare the baseline and revised flight 
control laws. A follow-on paper [9] describes a nonlinear robustness analysis method used to analyze the F/A-18 flight control laws.

The paper has the following structure. First, a six-DOF mathematical model is derived for the F/A-18 Hornet aircraft. The aerodynamic model is represented as closed-form polynomial expressions, with functional dependence on the states. The fallingleaf motion is described and simulation responses are presented showing the six-DOF F/A-18 aircraft model captures the falling-leaf characteristics. A simplified architecture of both the baseline and the revised flight control law are presented in Sec. III. Linear models suitable to analyze the falling-leaf motion are derived. Both the closed-loop linear models are analyzed using classical techniques and linear robustness concepts such as the structured singular value $(\mu)$ and worst-case analysis in Sec. IV. The paper concludes with a summary of the linear analysis of the F/A-18 controllers and the nonlinear aircraft model.

\section{F/A-18 Aircraft Description and Model Development}

A nonlinear mathematical model of the F/A-18 Hornet aircraft including its aerodynamic characteristics and control surface description is presented for the purpose of linear and nonlinear analysis of flight control system. The model is developed based on the publicly available aerodynamic data for the F/A-18 High-Alpha Research Vehicle (HARV) [3-ㅜㅇ. However, the derived model has limitations. First, the aerodynamic data of the F/A-18 HARV have been fitted as polynomial functions of angle-of-attack to be used in the model. Because of this polynomial fitting, it is possible to have disagreement with the fitted data and the real flight test data at certain region of the state-space. Second, due to lack of available aerodynamic data, the sideslip dependence of the aerodynamic characteristics of the aircraft is modeled with a simplified approach without incorporating any sideslip data and is explained later. This is unfortunate as it is known that the sideslip characteristics are an important aspect of the falling-leaf motion [1]. The proposed nonlinear aircraft model contains many of the nonlinear characteristics of the F/A-18 aircraft and is able to reproduce the falling-leaf motion. Hence, the model is sufficiently accurate to be used for the linear analysis of the flight controllers. Moreover, the MATLAB $\mathrm{M}$-files to generate the models and the results shown in this paper can be found elsewhere [10].

\section{A. F/A-18 Hornet Vehicle Description}

The U.S. Navy F/A-18 aircraft, Fig. $\underline{1}, \underline{\S}$ is a high-performance, twin engine fighter aircraft built by the McDonnell Douglas (currently the Boeing Company) Corporation. The F/A-18-A/B and F/A-18-C/D are single and two seat aircraft, respectively. These variants are commonly known as Hornet. Each engine of the Hornet is a General Electric, F404-GE-400 rated at 16,100-lbf of static thrust at sea level. The aircraft features a low sweep trapezoidal wing planform with $400 \mathrm{ft}^{2}$ area and twin vertical tails [11]. Table 1 lists the aerodynamic reference and physical parameters of the F/A-18 Hornet [11].

\section{B. Control Surfaces}

A conventional F/A-18 Hornet aircraft has five pairs of control surfaces: stabilators, rudders, ailerons, leading-edge flaps (LEF), and trailing-edge flaps (TEF). The LEFs and TEFs are used mostly during takeoff and landing. Hence, these control effectors are not considered in the control analysis and modeling. Only the symmetric stabilator, aileron and rudder are considered as control effectors for the analyses performed in this paper. Note that the differential stabilator's contribution in roll axis control is ignored in this paper for simplicity purpose. Longitudinal control or pitch axis control is provided by the symmetric deflection of the stabilators. Deflection of the ailerons is used to control the roll axis or lateral direction, and deflection of the

${ }^{\S}$ Data available online at http://www.dfrc.nasa.gov/Gallery/Photo/ F-18Chase/Small/EC02-0224-1.jpg [retrieved 01 May 2010].

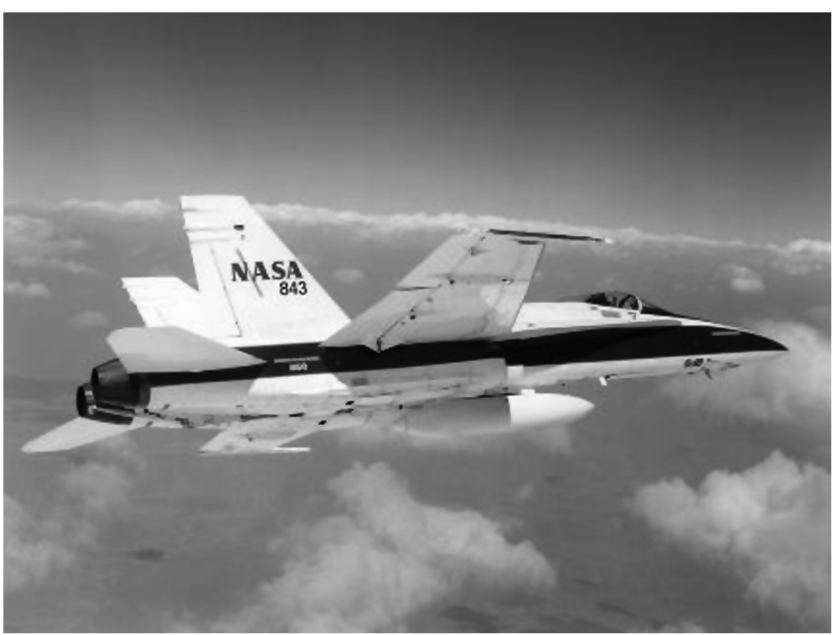

Fig. 1 F/A-18 Hornet.

rudders provides directional or yaw axis control. The hydraulic actuation systems for these primary controls are modeled as firstorder lags. Table 2 provides the mathematical models of the actuators and their deflection and rate limits [11].

\section{Aerodynamic Model}

The aircraft motion depends on the aerodynamic forces and moments acting on the vehicle. The aerodynamic forces consist of lift force ( $L$ in lbs), drag force ( $D$ in lbs), and sideforce ( $Y$ in lbs). The aerodynamic moments are described by the pitching moment ( $M$ in $\mathrm{ft}-\mathrm{lbs}$ ), rolling moment ( $l$ in ft-lbs), and yawing moment ( $n$ in ft-lbs). The aerodynamic forces and moments depend on the aerodynamic angles ( $\alpha$ and $\beta$ in rad), angular rates ( $p, q, r$ in $\mathrm{rad} / \mathrm{s}$ ) and control surface deflections $\left(\delta_{\text {stab }}, \delta_{\text {ail }}, \delta_{\text {rud }}\right.$ in rad $)$. These forces and moments are given by

$$
\begin{aligned}
D & =\bar{q} S C_{D}\left(\alpha, \beta, \delta_{\text {stab }}\right) \\
L & =\bar{q} S C_{L}\left(\alpha, \beta, \delta_{\text {stab }}\right)
\end{aligned}
$$

$$
Y=\bar{q} S C_{Y}\left(\alpha, \beta, \delta_{\text {ail }}, \delta_{\text {rud }}\right)
$$

$$
l=\bar{q} S b C_{l}\left(\alpha, \beta, \delta_{\text {ail }}, \delta_{\text {rud }}, p, r, V\right)
$$

Table 1 Aircraft parameters

\begin{tabular}{lcc}
\hline \hline Parameter & Symbol & Value \\
\hline Wing area & $S$ & $400 \mathrm{ft}^{2}$ \\
Mean aerodynamic chord & $\bar{c}$ & $11.52 \mathrm{ft}$ \\
Wing span & $b$ & $37.42 \mathrm{ft}$ \\
Mass & $m$ & $1034.5 \mathrm{slug}$ \\
Roll axis moment of inertia & $I x x$ & $23,000 \mathrm{slug} \cdot \mathrm{ft}^{2}$ \\
Pitch axis moment of inertia & $I_{y y}$ & $151,293 \mathrm{slug} \cdot \mathrm{ft}^{2}$ \\
Yaw axis moment of inertia & $I_{z z}$ & $169,945 \mathrm{slug} \cdot \mathrm{ft}^{2}$ \\
Cross product of inertia about y axis & $I_{x z}$ & $-2971 \mathrm{slug} \cdot \mathrm{ft}^{2}$ \\
\hline \hline
\end{tabular}

Table 2 Control surface and actuator configuration

\begin{tabular}{lclc}
\hline \hline Actuator & Rate limit & Position limit & Model \\
\hline Stabilator, $\delta_{\text {stab }}$ & $\pm 40^{\circ} / \mathrm{s}$ & $-24^{\circ},+10.5^{\circ}$ & $\frac{30}{s+30}$ \\
Aileron, $\delta_{\text {ail }}$ & $\pm 100^{\circ} / \mathrm{s}$ & $-25^{\circ},+45^{\circ}$ & $\frac{48}{s+48}$ \\
Rudder, $\delta_{\text {rud }}$ & $\pm 61^{\circ} / \mathrm{s}$ & $-30^{\circ},+30^{\circ}$ & $\frac{40}{s+40}$ \\
\hline \hline
\end{tabular}




$$
M=\bar{q} S \bar{c} C_{M}\left(\alpha, \delta_{\text {elev }}, q, V\right)
$$

$$
n=\bar{q} S b C_{n}\left(\alpha, \beta, \delta_{\text {ail }}, \delta_{\text {rud }}, p, r, V\right)
$$

where $\bar{q}:=\frac{1}{2} \rho V^{2}$ is the dynamic pressure $\left(\mathrm{lbs} / \mathrm{ft}^{2}\right)$ and $\rho$ is the air density $\left(\mathrm{lbs} / \mathrm{ft}^{3}\right) . C_{D}, C_{L}, C_{Y}, C_{l}, C_{M}$, and $C_{n}$ are unitless aerodynamic coefficients. Tables $\mathrm{A} 1$ and $\mathrm{A} 2$ provide the data for the aerodynamic coefficients of the F/A-18 model used in this paper. The functional form for the aerodynamic coefficients can be expressed as a sum of terms that model the aerodynamic effects of the basic airframe $\left(C_{*}\right.$, basic), control inputs $\left(C_{*}\right.$, control) and angular rate damping $\left(C_{*}\right.$, rate). Here, $C_{*}$ can be replaced by $C_{D}, C_{L}, C_{Y}, C_{l}, C_{M}$, $C_{n}$ :

$$
\begin{aligned}
& C_{D}\left(\alpha, \beta, \delta_{\text {stab }}\right)=C_{D, \text { basic }}(\alpha, \beta)+C_{D, \text { control }}\left(\alpha, \delta_{\text {stab }}\right) \\
& C_{L}\left(\alpha, \beta, \delta_{\text {stab }}\right)=C_{L, \text { basic }}(\alpha, \beta)+C_{L, \text { control }}\left(\alpha, \delta_{\text {stab }}\right) \\
& C_{Y}\left(\alpha, \beta, \delta_{\text {ail }}, \delta_{\text {rud }}\right)=C_{Y, \text { basic }}(\alpha, \beta)+C_{Y, \text { control }}\left(\alpha, \delta_{\text {rud }}, \delta_{\text {ail }}\right) \\
& C_{l}\left(\alpha, \beta, V, \delta_{\text {ail }}, \delta_{\text {rud }}\right)=C_{l, \text { basic }}(\alpha, \beta)+C_{l, \text { control }}\left(\alpha, \delta_{\text {rud }}, \delta_{\text {ail }}\right) \\
& +C_{l, \text { rate }}(\alpha, p, r, V) \\
& C_{M}\left(\alpha, \beta, V, \delta_{\text {stab }}\right)=C_{M, \text { basic }}(\alpha)+C_{M, \text { control }}\left(\alpha, \delta_{\text {stab }}\right) \\
& +C_{M, \text { rate }}(\alpha, q, V) \\
& C_{n}\left(\alpha, \beta, V, \delta_{\text {ail }}, \delta_{\text {rud }}\right)=C_{n, \text { basic }}(\alpha, \beta)+C_{n, \text { control }}\left(\alpha, \delta_{\text {rud }}, \delta_{\text {ail }}\right) \\
& +C_{n, \text { rate }}(\alpha, p, r, V)
\end{aligned}
$$

Because of lack of available data, the rate damping effect on the aerodynamic force coefficients $\left(C_{D}, C_{L}, C_{Y}\right)$ is ignored in the model formulation. These force coefficients are modeled based only on contributions from the basic airframe and control surfaces.

A number of publications are available which provide flight test data for the stability and control derivatives of the F/A-18 HARV vehicle [3-8], which has similar aerodynamic characteristics to the F/ A-18 Hornet aircraft [12]. The aerodynamic data of the F/A-18 HARV is used to formulate the aerodynamic model for the F/A-18 Hornet. Least-square fitting of the flight data [3-8] is performed to derive a closed-form expression to the aerodynamic model. Section I of the Appendix provides the least squares fits for the aerodynamic coefficients.

Two important issues arise with fitting the aerodynamic coefficients. First, the flight test data are provided over a range of 5 or 10 to $60^{\circ}$ angle-of-attack with fewer data points at low angle-ofattack $\left(0^{\circ} \leq \alpha \leq 10^{\circ}\right)$. Extrapolation of data within the lower range of angle-of-attack can lead to unrealistic fit which may lead to unrealistic aerodynamic characteristics at low angle-of-attack. For traditional aircraft, the aerodynamic characteristics of the vehicle do not change significantly at low angle-of-attack $\left(\alpha \leq 10^{\circ}\right)$. Hence if data is unavailable, the aerodynamic coefficient is held constant for angle-of-attack between 0 and $10^{\circ} . \mathbb{1}$ The resulting model is valid for an angle-of-attack range from $0-60^{\circ}$. Second, data is unavailable for nonzero sideslip flight conditions. However, the basic airframe coefficients are functionally dependent on both $\alpha, \beta$. In this paper, the basic airframe dependence of $C_{Y}, C_{l}, C_{n}$, respectively $C_{Y \text {,basic }}(\alpha, \beta)$, $C_{l, \text { basic }}(\alpha, \beta), C_{n \text {,basic }}(\alpha, \beta)$, in Eq. $(2 \mathrm{c}),(2 \mathrm{~d})$, and $(2 \mathrm{f})$, are approximated as $C_{Y \text {,basic }}(\alpha) \beta, C_{l \text {,basic }}(\alpha) \bar{\beta}, C_{n \text {,basic }}(\alpha) \beta$ to account for this lack of data. This indicates, for $C_{Y}$, that the sideforce is

\footnotetext{
'Personal communication with Dr. John V. Foster, NASA Langley Research Center.
}

expected to be zero when the sideslip is zero. This approximation step can also be viewed as linearization of the sideslip effect around the origin. Similar approach has been shown in the book by Stevens and Lewis [13]. Moreover, analytical expressions of $C_{D \text {,basic }}(\alpha, \beta)$, $C_{L, \text { basic }}(\alpha, \beta)$ are extracted from []].

\section{Equations of Motion}

A six-DOF nine-state mathematical model for the F/A-18 aircraft is presented in this section only for completeness and notational simplicity. A more detailed description of the conventional aircraft equations of motion is provided in $[\underline{7}, \underline{14}, \underline{15}]$. The equations of motion take the form

$$
\dot{x}=f(x, u)
$$

where $\quad x:=[V(\mathrm{ft} / \mathrm{s}), \beta(\mathrm{rad}), \alpha(\mathrm{rad}), p(\mathrm{rad} / \mathrm{s}), q(\mathrm{rad} / \mathrm{s}) r(\mathrm{rad} / \mathrm{s})$, $\phi(\mathrm{rad}), \theta(\mathrm{rad}), \psi(\mathrm{rad})], \quad$ and $\quad u:=\left[\delta_{\mathrm{ail}}(\mathrm{rad}), \delta_{\mathrm{rud}}(\mathrm{rad}), \delta_{\mathrm{stab}}(\mathrm{rad})\right.$, $T(\mathrm{lbf})]$. Note that the aerodynamic model and the equations of motion are presented in the units of radians. However, for ease of interpretation, results are presented in the units of degree in this paper.

\section{Euler Angles}

The rate of change of aircraft's angular positions are provided in Eq. (4):

$$
\left[\begin{array}{c}
\dot{\phi} \\
\dot{\theta} \\
\dot{\psi}
\end{array}\right]=\left[\begin{array}{ccc}
1 & \sin \phi \tan \theta & \cos \phi \tan \theta \\
0 & \cos \phi & -\sin \phi \\
0 & \sin \phi \sec \theta & \cos \phi \sec \theta
\end{array}\right]\left[\begin{array}{l}
p \\
q \\
r
\end{array}\right]
$$

\section{Force Equations}

The aerodynamic forces, gravity forces and thrust force applied to the aircraft are considered. Thrust force is assumed to be constant. Equation (5) defines the force equations:

$$
\begin{aligned}
\dot{V}= & -\frac{1}{m}(D \cos \beta-Y \sin \beta)+g(\cos \phi \cos \theta \sin \alpha \cos \beta \\
+ & \sin \phi \cos \theta \sin \beta \quad-\sin \theta \cos \alpha \cos \beta)+\frac{T}{m} \cos \alpha \cos \beta \\
\dot{\alpha}= & -\frac{1}{m V \cos \beta} L+q-\tan \beta(p \cos \alpha+r \sin \alpha) \\
& +\frac{g}{V \cos \beta}(\cos \phi \cos \theta \cos \alpha+\sin \alpha \sin \theta)-\frac{T \sin \alpha}{m V \cos \beta} \\
\dot{\beta}= & \frac{1}{m V}(Y \cos \beta+D \sin \beta)+p \sin \alpha-r \cos \alpha \\
& +\frac{g}{V} \cos \beta \sin \phi \cos \theta \\
& +\frac{\sin \beta}{V}\left(g \cos \alpha \sin \theta-g \sin \alpha \cos \phi \cos \theta+\frac{T}{m} \cos \alpha\right)
\end{aligned}
$$

\section{Moment Equations}

The aerodynamic moments are associated with external applied moments. The gyroscopic effect of the moment is neglected. Equation (6) describes the corresponding moment equations for the F/A-18 Hornet: 


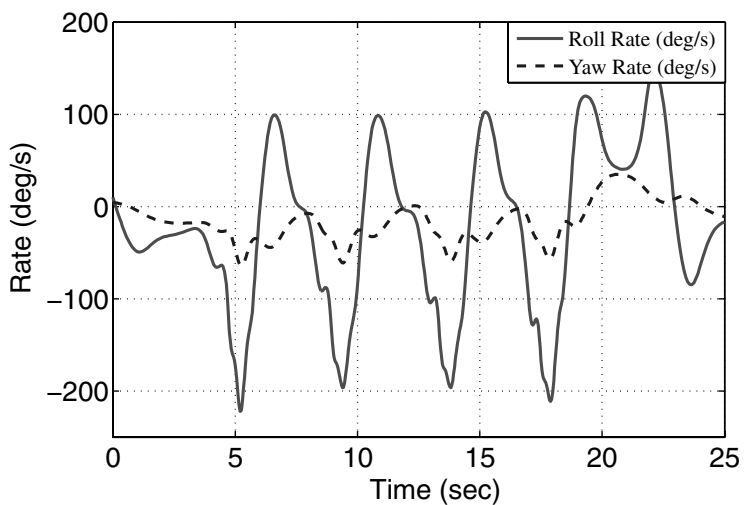

Fig. 2 Large and coupled roll rate, yaw rate oscillation during fallingleaf motion.

$$
\begin{aligned}
& {\left[\begin{array}{c}
\dot{p} \\
\dot{q} \\
\dot{r}
\end{array}\right]=\left[\begin{array}{ccc}
\frac{I_{z z}}{\kappa} & 0 & \frac{I_{x z}}{\kappa} \\
0 & \frac{1}{I_{y y}} & 0 \\
\frac{I_{x z}}{\kappa} & 0 & \frac{I_{x x}}{\kappa}
\end{array}\right]\left(\left[\begin{array}{c}
l \\
M \\
n
\end{array}\right]\right.} \\
&\left.-\left[\begin{array}{ccc}
0 & -r & q \\
r & 0 & -p \\
-q & p & 0
\end{array}\right]\left[\begin{array}{ccc}
I_{x x} & 0 & -I_{x z} \\
0 & I_{y y} & 0 \\
-I_{x z} & 0 & I_{z z}
\end{array}\right]\left[\begin{array}{c}
p \\
q \\
r
\end{array}\right]\right)
\end{aligned}
$$

where $\kappa=I_{x x} I_{z z}-I_{x z}^{2}$.

Equations (4-6) describe the mathematical model for the F/A-18 aircraft with the aerodynamic model presented in Sec. II.C. This is a standard mathematical representation for describing aircraft dynamics.

\section{E. Falling-Leaf}

The original F/A-18A/B/C/D Hornet aircraft are susceptible to an out-of-control flight departure phenomenon with sustained oscillation, namely 'falling-leaf' motion. The falling-leaf mode is briefly described in the following paragraphs. A more detailed discussion of the falling-leaf motion can be found in other [1,2].

The falling-leaf motion of an aircraft can be characterized as large, coupled out-of-control oscillations in the roll rate $(p)$ and yaw rate $(r)$ direction combined with large fluctuations in angle-of-attack $(\alpha)$ and sideslip $(\beta)[1,2]$. Figure 2 shows the typical roll rate and yaw rate trajectories associated with the falling-leaf motion [1,2]. This out-ofcontrol mode exhibits periodic in-phase roll and yaw rates with large amplitude fluctuations about small or zero mean. Generation of the roll and yaw rate responses are mainly due to the large sideslip oscillation. During large sideslip and angle-of-attack motion, the dihedral effect (roll caused by sideslip) of the aircraft wings becomes extremely large and the directional stability becomes unstable. The like-signs of these two components are responsible for the in-phase

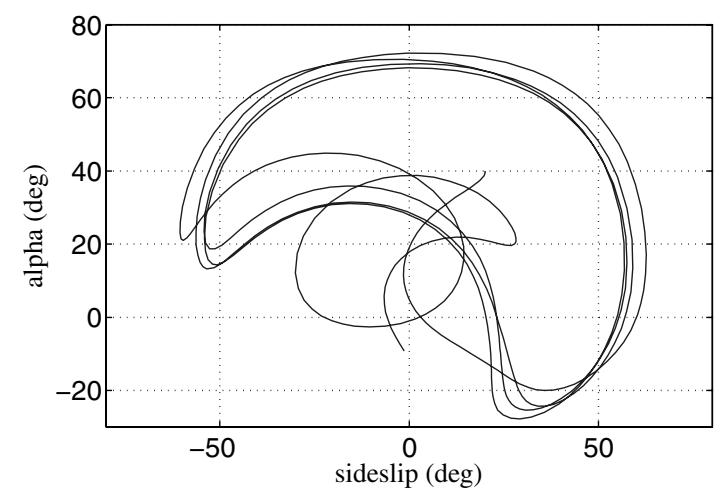

Fig. 3 Time response of $\alpha$ vs $\beta$ produces a mushroom shape curve during falling-leaf motion.
Table 3 Falling-leaf categories

\begin{tabular}{lccc}
\hline \hline State & Slow mode & Fast mode & High $\alpha$ mode \\
\hline$\alpha, \mathrm{deg}$ & -5 to +60 & +20 to +70 & +30 to +85 \\
$\beta, \mathrm{deg}$ & -40 to +40 & -40 to +40 & -40 to +40 \\
$p, \mathrm{deg} / \mathrm{s}$ & -120 to +150 & -90 to +130 & -90 to +130 \\
$r, \mathrm{deg} / \mathrm{s}$ & -50 to +50 & -10 to +60 & -10 to +75 \\
Period, $\mathrm{s}$ & 7 & 4.7 & 4.5 \\
Frequency, rad/s & 0.898 & 1.34 & 1.39 \\
\hline \hline
\end{tabular}

motion. The roll rate motion can easily reach up to $\pm 120^{\circ} / \mathrm{s}$, while the yaw rate motion can fluctuate around $\pm 50^{\circ} / \mathrm{s}$. During this motion, the value of angle-of-attack can reach up to $\pm 70^{\circ}$ with sideslip oscillations between $\pm 40^{\circ}$ [1]. The required aerodynamic nose-down pitching moment is exceeded by the pitch rate generation due to the inertial coupling of the in-phase roll and yaw rates. The reduction in pitching moment is followed by a reduction in normal force, eventually causing a loss of lift in the aircraft. Another distinguishing feature of the falling-leaf motion is the time response of $\alpha$ vs $\beta$ produces a mushroom shape curve as shown in Fig. 3 .

The characteristics of the falling-leaf motion are nonlinear in nature. Figures 2 and 3 are generated by simulating the nonlinear $\mathrm{F} /$ A-18 model presented in this paper. The falling-leaf motion shown in Figs. $\underline{2}$ and $\underline{3}$ are generated with the following initial condition:

$$
x_{o}:=\left[350(\mathrm{ft} / \mathrm{s}) 20^{\circ} 40^{\circ} 10^{\circ} / \mathrm{s} 0^{\circ} / \mathrm{s}^{\circ} / \mathrm{s}^{\circ} 0^{\circ} 0^{\circ}\right]
$$

Note that units of degree are used for ease of interpretation. The ordering of the states $x$ are same as mention in Eq. (3). The inputs are set to zeros for this particular simulation. Note that the falling-leaf responses cannot be generated by simulating the linearized models as described later in Section IV.A.

A study of the falling-leaf motion [1] has categorized the motion into three different spectrum: 1) slow falling-leaf, 2) fast falling-leaf, and 3) high-alpha fast falling-leaf. The primary differences in shifting from the slow to the fast mode can be contributed to the movement of $\alpha$ to higher values, the biasing of yaw rate, and an increase in the frequency of the oscillation. Table 3 categorizes the three falling-leaf motion as reported in the study [1] $]$. The analyses presented in this paper are closely related to the slow falling-leaf mode category.

\section{F. Open-Loop Validation of the Developed F/A-18 Model}

The developed F/A-18 model is validated against flight test data of the F/A-18 HARV available in the report by Iliff and Wang [16]. Figure 8 from the report by Iliff and Wang [16] showed flight test results for a specific longitudinal maneuver. The maneuver is performed by exciting symmetric aileron, trailing-edge-flap and stabilator. However, the control surfaces are excited independently. Hence, the comparison is performed for the time duration when the stabilator channel is excited, which is $10-15 \mathrm{~s}$ (see Fig. 8 from Iliff and Wang [16]). Figure $\underline{4}$ shows the comparison of the state responses. The stabilator input signal (lower right subplot of Fig. 1) was reconstructed from the data shown in Iliff and Wang [16]. The proposed F/A-18 model is simulated using this reconstructed input signal. The simulation responses are shown in dashed line. The flight test results are marked as " $\mathrm{x}$ " and are reproduced in this paper. Notice that the flight condition of the simulation model $\left(V_{t}=335 \mathrm{ft} / \mathrm{s}\right.$, $\left.\alpha_{t}=19^{\circ}\right)$ is slightly different than the flight test results $\left(V_{t}=\right.$ $400 \mathrm{ft} / \mathrm{s}, \alpha_{t}=24^{\circ}$ ) [16]. However, the state responses are qualitatively very similar for the given excitation in the stabilator channel. For example, in both cases, the angle-of-attack comes down by $6^{\circ}$ from its own trim point around $2.5 \mathrm{~s}$. Then the angle-of-attack goes up by roughly $8^{\circ}$ around $4.5 \mathrm{~s}$. Likewise, qualitative characteristics are very similar in other state responses also. The lateral-directional states are not plotted because they are not excited by the stabilator channel. 

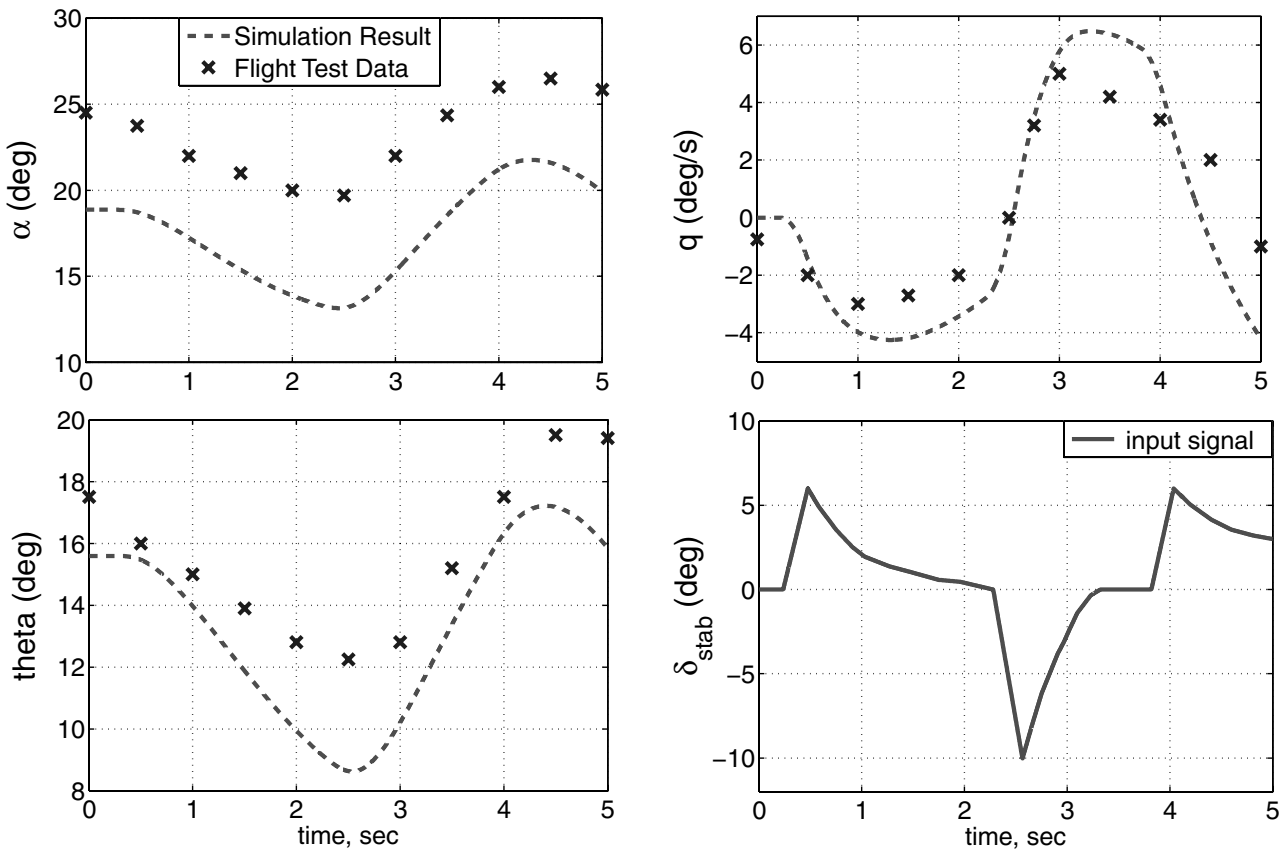

Fig. 4 Comparison of open-loop simulation model and flight test results from Iliff and Wang [16] due to a perturbation in the stabilator channel.

\section{F/A-18 Flight Control Laws}

The simplified architecture of the baseline flight control and the revised flight control law is presented in Secs. III.A and III.B, respectively. This control law structure represents the stability augmentation system of the Hornet flight control system. Simplification of the F/A-18 flight control system was deemed appropriate because the paper is only concerned with the stability robustness of the F/A18 aircraft. Section II of the Appendix provides the state-space realization for both control laws.

\section{A. Baseline Control Law}

Figure 5 shows the control law architecture for the baseline control laws used for analysis in this paper. The baseline controller structure for the F/A-18 aircraft closely follows the control augmentation system presented in the report by Buttrill et al. [11]. The actuators are modeled as a first-order lag, as mentioned in Table $\underline{2}$.

\section{Longitudinal Control}

The longitudinal baseline control design for the F/A-18 aircraft includes angle-of-attack ( $\alpha$ in rad), normal acceleration $\left(a_{n}\right.$ in $\left.\mathrm{g}\right)$, and

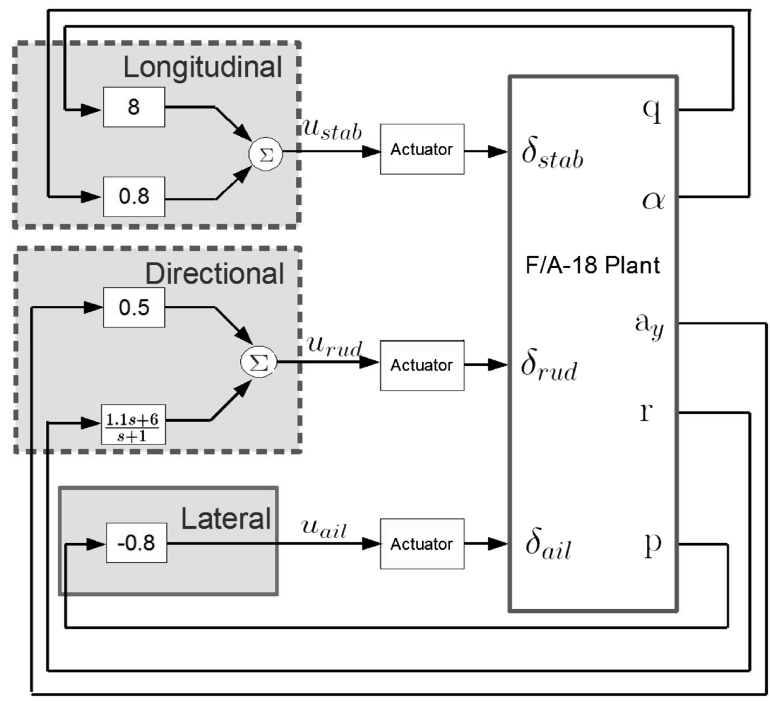

Fig. 5 F/A-18 baseline flight control law. pitch rate ( $q$ in $\mathrm{rad} / \mathrm{s}$ ) measurement feedback. The angle-of-attack feedback is used to stabilize an unstable short-period mode that occurs during low-speed, high-angle-of-attack maneuvering. The inner-loop pitch rate feedback is composed of a proportional feedback gain, to improve damping of the short-period mode. In the high speed regime, the pitch rate proportional gain needs to be increased to avoid any unstable short-period mode.

The normal acceleration feedback, a proportional-integrator compensator, is not implemented in the simplified control law structure. The feedback gain of the normal acceleration is significantly lower at the flight condition of interest than that of alpha feedback gain. In addition, the values of normal acceleration around the flight condition of interest are estimated by performing numerous simulations. The estimated value is bounded by $1.22 \mathrm{~g}$. Hence, the contribution of the normal acceleration feedback term is negligible and eliminated from the analysis.

\section{Lateral Control}

Control of the lateral direction axis involves roll rate $(p)$ feedback to the aileron actuators. Roll rate feedback is used to improve roll

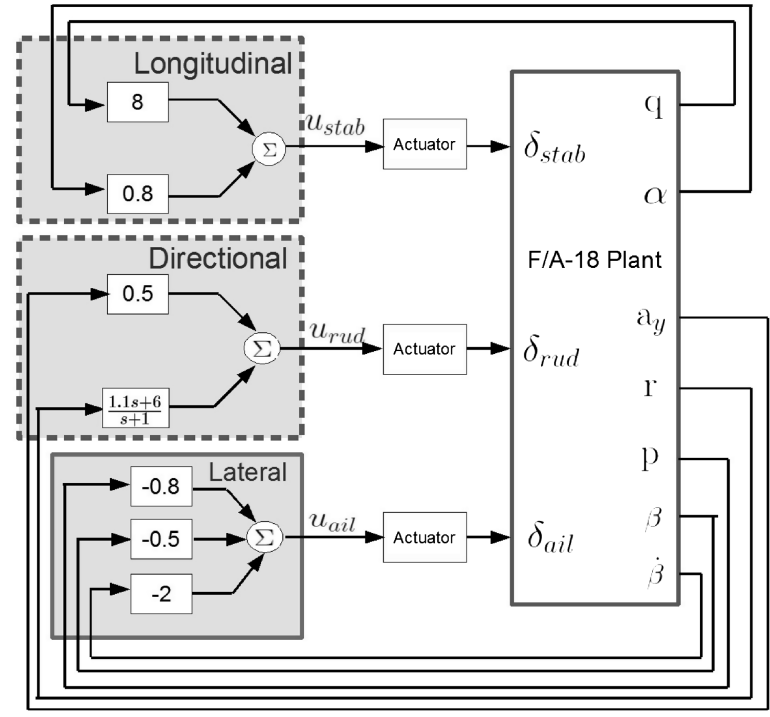

Fig. 6 F/A-18 revised flight control law. 
Table 4 Trim values around $V_{t}=350 \frac{\mathrm{ft}}{\mathrm{s}}$, altitude $=25,000 \mathrm{ft}$

\begin{tabular}{lcccccccc}
\hline \hline State/input & Plant 1 & Plant 2 & Plant 3 & Plant 4 & Plant 5 & Plant 6 & Plant 7 & Plant 8 \\
\hline$\alpha_{t}$, deg & 15.29 & 15.59 & 17.43 & 20.29 & 15.59 & 16.16 & 18.41 & 21.40 \\
$\beta_{t}, \mathrm{deg}$ & 0 & 0 & 0 & 0 & 10 & 10 & 10 & 10 \\
$\phi_{t}, \mathrm{deg}$ & 0 & 10 & 25 & 35 & 0 & 10 & 25 & 35 \\
$p_{t}, \mathrm{deg} / \mathrm{s}$ & 0 & 0.1322 & 0.8695 & 1.845 & -0.1478 & -0.5188 & -1.074 & -1.353 \\
$r_{t}, \mathrm{deg} / \mathrm{s}$ & 0 & 0.7500 & 1.864 & 2.635 & 0.3276 & 1.084 & 2.141 & 2.821 \\
$q_{t}, \mathrm{deg} / \mathrm{s}$ & 0 & 0.1322 & 0.8695 & 1.845 & 0 & 0.1911 & 0.9982 & 1.975 \\
$\theta_{t}, \mathrm{deg}$ & 26.10 & 25.67 & 22.98 & 18.69 & 24.27 & 25.24 & 24.45 & 21.45 \\
$\psi_{t}, \mathrm{deg}$ & 0 & 0 & 0 & 0 & 0 & 0 & 0 & 0 \\
$\delta_{\mathrm{Stab}_{t}}, \mathrm{deg}$ & -2.606 & -2.683 & -3.253 & -4.503 & -2.669 & -2.823 & -3.606 & -5.101 \\
$\delta_{\text {Ail }_{t}, \mathrm{deg}}$ & 0 & -0.1251 & -0.3145 & -0.4399 & 12.21 & 12.45 & 13.72 & 15.60 \\
$\delta_{\mathrm{Rud}_{1}}, \mathrm{deg}$ & 0 & -0.3570 & -0.9109 & -1.359 & 13.24 & 12.73 & 11.22 & 8.334 \\
$T_{t}, \mathrm{lbf}$ & 14,500 & 14,500 & 14,500 & 14,500 & 14,500 & 14,500 & 14,500 & 14,500 \\
\hline \hline
\end{tabular}

damping and the roll-subsidence mode of the aircraft. Because of the inherent high roll damping associated with the F/A-18 aircraft at high speed, the roll rate feedback authority is usually reduced at high dynamic pressure. In the low speed regime, the roll rate feedback gain is used to increase damping of the Dutch-roll mode. The roll rate feedback gain ranges between 0.8 at low speed to 0.08 at high speed. The flight conditions considered in the analysis in this paper are at a forward velocity of $350 \mathrm{ft} / \mathrm{s}$. At this speed, a feedback gain of 0.8 is used to provide roll damping.

\section{Directional Control}

Directional control involves feedback from yaw rate $(r)$ and lateral acceleration $\left(a_{y}\right)$ to the rudder actuators. Yaw rate is fed back to the rudder to generate a yawing moment. Yaw rate feedback reduces the yaw rate contribution to the Dutch-roll mode. In a steady state turn, there is always a constant nonzero yaw rate present. This requires the pilot to apply larger than normal rudder input to negate the effect of the yaw damper and make a coordinated turn. A washout filter is used to effectively eliminate this effect. The filter approximately differentiates the yaw rate feedback signal at low frequency, effectively eliminating yaw rate feedback at steady state conditions [13]. The lateral acceleration feedback contributes to reduce sideslip during turn coordination.

\section{B. Revised Control Law}

Figure 6 shows the architecture of the revised F/A-18 flight control law as described in the papers by Heller et al. [2,17]. The objective of the revised flight control law was to improve the departure resistance characteristics and full recoverability of the F/A-18 aircraft without sacrificing the maneuverability of the aircraft [2]. The significant changes in the revised control law are the additional sideslip $(\beta)$ and sideslip rate $(\dot{\beta})$ feedback to the aileron and the differential stabilator channels [2]. Note, the differential stabilator channel is ignored in this paper. Both these channels are useful to control sideslip with the ailerons being most effective to roll the aircraft at high angles-ofattack and the differential stabilator in aiding the vehicle to yaw. Since mainly roll maneuvers with small sideslip angle are considered in this paper, the differential stabilator too was deemed appropriate to ignore.

There are no direct measurements of sideslip and sideslip rate. The sideslip and the sideslip rate feedback signals are computed based on already available signals from the sensors and using the kinematics of the aircraft. Specifically, sideslip rate $(\dot{\beta})$ is estimated by using a firstorder approximation to the sideslip state derivative equation. The sideslip feedback plays a key role in increasing the lateral stability in the $30-35^{\circ}$ range of angle-of-attack. The sideslip rate feedback improves the lateral-directional damping. Hence, sideslip motion is damped even at high angles-of-attack. This feature is key to eliminating the falling-leaf mode behavior of the aircraft, which is an aggressive form of in-phase Dutch-roll motion. Proportional feedback is implemented in these two feedback channels. The values of the proportional gains are $k_{\beta}=0.5$ and $k_{\dot{\beta}}=2$.

\section{Linear Analysis}

Current practice for validating flight control laws relies on applying linear analysis tools to assess the closed-loop stability and performance characteristics about many trim conditions. Linear analysis investigates robustness issues and possibly worst-case scenarios around the operating points. In this paper, the F/A-18 aircraft is trimmed around different operating points of interest that are suitable to characterize the falling-leaf motion. A reduced sixstate linear representation is extracted from the nine-state linear models around these operating points. This six-state linear representation is used to construct the closed-loop models for both the baseline and revised flight control law for linear robustness analysis.

\section{A. Linear Model Formulation}

Linear models are formulated around selected operating points. The flight conditions for these operating points are chosen such that the aircraft is likely to experience the falling-leaf motion. Sec. II.F characterized the falling-leaf motion similar to the aggressive Dutchroll motion with strong coupling in all three axes: longitudinal, lateral and directional. Flight conditions that exhibit coupling in all three directions are suitable candidates for analyzing the falling-leaf motion. Bank angle maneuvers exhibit coupling in all three directions are used to analyze the falling-leaf motion. Both coordinated $(\beta=0 \mathrm{deg})$ and uncoordinated $\left(\beta \neq 0^{\circ}\right)$ turns with 0 , 10,25 , and $35^{\circ}$ bank angle are considered. Simulation results [1] have shown the velocity of the aircraft is usually within $250-350 \mathrm{ft} / \mathrm{s}$ during the falling-leaf motion. The F/A-18 aircraft is trimmed around $V_{t}=350 \mathrm{ft} / \mathrm{s}$. Table $\underline{4}$ provides the trim values for the flight conditions considered in this paper. The subscript $t$ denotes the trim value.

The F/A-18 aircraft is linearized around the trim points specified in Table 4 . The linearized plants have the following form:

$$
\begin{aligned}
& \dot{x}=A x+B u \\
& y=C x+D u
\end{aligned}
$$

Table 5 Comparison of flight conditions and eigenvalues in the longitudinal direction between models from current paper and models available in the literature [18]

\begin{tabular}{lcc}
\hline \hline Mode & Current paper & Referenced paper [18] \\
\hline Condition 1 $\left(V_{t}, \alpha_{t}\right)$ & $\left(368 \mathrm{ft} / \mathrm{s}, 20^{\circ}\right)$ & $\left(368 \mathrm{ft} / \mathrm{s}, 15^{\circ}\right)$ \\
Eigenvalues 1 & & \\
$\quad$ Short period & $-0.221 \pm 0.570 i$ & $-0.197 \pm 0.628 i$ \\
$\quad$ Phugoid & $-0.0109 \pm 0.115 i$ & $-0.00957 \pm 0.123 i$ \\
Condition 2 $\left(V_{t}, \alpha_{t}\right)$ & $\left(290 \mathrm{ft} / \mathrm{s}, 30^{\circ}\right)$ & $\left(290 \mathrm{ft} / \mathrm{s}, 35^{\circ}\right)$ \\
Eigenvalues 2 & & \\
$\quad$ Short period & $-0.153 \pm 1.15 i$ & $-0.190 \pm 0.558 i$ \\
$\quad$ Phugoid & $-0.0614 \pm 0.138 i$ & $-0.0375 \pm 0.147 i$ \\
\hline \hline
\end{tabular}


where $x, u$ are described in Section II.D and $y$ denotes the output, $y:=\left[\begin{array}{lllllll}a_{y}, & p, & r & \alpha, & \beta & q & \dot{\beta}\end{array}\right]$. Recall, $a_{y}=\frac{\bar{q} S}{m g} C_{y}$ and $\dot{\beta}$ is computed based on already available signals from the sensors and using the kinematics of the aircraft. The linearized equations for both $a_{y}$ and $\dot{\beta}$ are used as output signals in this paper. Section III of the Appendix provides the linear state-space data for plant 4 and plant 8 presented in Table 4.

The eigenvalues of the linear models generated from the proposed F/A-18 model are compared with the eigenvalues of the F/A-18 HARV available in the literature [18,19]. Table 5 provides comparison of the eigenvalues and flight conditions for the longitudinal direction at steady-level flight. Two flight conditions are considered from the paper by Ostroff et al. [18]. Note that the trim condition $\alpha_{t}$ produced by the model in this paper differs from the trim condition reported in the paper by Ostroff et al. [18] by $5^{\circ}$. The longitudinal axis eigenvalues are very close for the linear model around $V_{T}=368 \mathrm{ft} / \mathrm{s}$, while the eigenvalues do not match for the plant around $V_{T}=290 \mathrm{ft} / \mathrm{s}$. This mismatch can be attributed to the polynomial modeling of the pitching moment curve around the trimmed angle-of-attack $\left(\alpha_{t}=30^{\circ}\right)$ at that flight condition. The lookup table pitching moment data [16] has a sharp drop in pitching moment values within $25^{\circ} \leq \alpha \leq 40^{\circ}$. The second-order polynomial fit to the pitching moment data, used in this paper, does not capture this characteristic of the lookup table data. Hence, the eigenvalues do not match with each other for the flight condition around $V_{T}=290 \mathrm{ft} / \mathrm{s}$.

Similarly, for the lateral axis, two airspeeds, $V_{t}=361308 \mathrm{ft} / \mathrm{s}$ are considered. The flight conditions and eigenvalues of the lateral direction are reported in the paper by Davidson et al. [19]. Table $\underline{6}$ compares the eigenvalues and flight conditions for the lateral direction at steady-level flight. In both the cases, the flight conditions and the eigenvalues achieve a good match. The three dynamic modes of the lateral direction have similar eigenvalues for both the models.

A six-state representation of the F/A-18 model is extracted from the preceding nine-state model, described in Eq. (8). Decoupling the three states $V, \theta, \psi$ from the nine-state linear model results in the following six-state model with the thrust input held constant at the trim value:

$$
\dot{x}_{6}=A x_{6}+B u_{3}
$$

Table 6 Comparison of flight conditions and eigenvalues in the lateral direction between models from current paper and models available in the literature [19]

\begin{tabular}{lcc}
\hline \hline Mode & Current paper & Referenced paper [19] \\
\hline Condition 1 $\left(V_{t}, \alpha_{t}\right)$ & $\left(361 \mathrm{ft} / \mathrm{s}, 15^{\circ}\right)$ & $\left(361 \mathrm{ft} / \mathrm{s}, 15^{\circ}\right)$ \\
Eigenvalues 1 & & \\
$\quad$ Dutch roll & $-0.222 \pm 1.50 i$ & $-0.162 \pm 1.76 i$ \\
$\quad$ Roll subsidence & -0.330 & -0.279 \\
$\quad$ Spiral & -0.0754 & -0.0323 \\
Condition 2 $\left(V_{t}, \alpha_{t}\right)$ & $\left(308 \mathrm{ft} / \mathrm{s}, 25^{\circ}\right)$ & $\left(308 \mathrm{ft} / \mathrm{s}, 25^{\circ}\right)$ \\
Eigenvalues 2 & & \\
$\quad$ Dutch roll & $-0.155 \pm 1.55 i$ & $-0.179 \pm 1.76 i$ \\
$\quad$ Roll subsidence & -0.244 & -0.213 \\
$\quad$ Spiral & -0.0199 & -0.121 \\
\hline \hline
\end{tabular}

$$
y=C x_{6}+D u_{3}
$$

where $\quad x_{6}:=\left[\begin{array}{llllll}\beta, & \alpha, & p, & q, & r, & \phi\end{array}\right] \quad$ and $\quad u_{3}:=$ $\left[\begin{array}{lll}\delta_{\text {ail }}, & \delta_{\text {rud }}, & \delta_{\text {stab }}\end{array}\right]$.

Table 7 compares the eigenvalues of the linear plant (plant 4) with nine-state and six-state representations. The zero eigenvalue in the nine-state linear plant is due to the heading angle $(\psi)$ state, which does not affect the dynamics. Moreover, the period of the phugoid mode $\left(T_{p} \approx 50.3 \mathrm{~s}\right)$, involving $V$ and $\theta$ states, is separated by more than an order of magnitude to the one of the short-period ( $T_{p} \approx 3.79 \mathrm{~s}$ ), as shown in Table $\underline{7}$. Hence, these two states can be ignored.

Figures 7 and 8 show a Bode plot of the magnitude and phase response for the nine-state and six-state model representation from the stabilator channel input to the six states $\left(x_{6}\right)$. The magnitude and phase plots show that the six-state approximation is a good approximation in the interested falling-leaf region, above $0.9 \mathrm{rad} / \mathrm{s}$. The two models differ in the low frequency $(\leq 0.9 \mathrm{rad} / \mathrm{s})$ region. The low frequency mismatch is deemed acceptable in terms of capturing the characteristics of the falling-leaf motion.

The lateral-directional modes are important to capture the in-phase roll-yaw oscillation characteristics of the falling-leaf motion. Hence, these dynamic modes (Dutch-roll, roll-subsidence and spiral mode) involving $\beta, p, r, \phi$ states are kept in the formulation of the linear plant. The longitudinal states $\alpha, q$ are also retained to capture the short-period mode. Table 7 provides the eigenvalue characteristics of the two linear representation. The reduced six-state representation retains the dynamic modes of the aircraft, excluding the phugoid mode.

Six-state linear models are constructed for each of the eight operating points (see Table 4). These six-state linear representations are used to construct the closed-loop model for the baseline and revised flight control law. Eight closed-loop systems are formulated for each of the flight control law; four plants for coordinated turn and four associated with an uncoordinated turn. A variety of linear robustness concepts are employed in Secs. IV.B-IV.D to compare the stability performance between the baseline and the revised flight control law.

\section{B. Loop Margin Analysis}

Gain and phase margins are classical measures of robustness for the closed-loop system. A typical requirement for certification of a flight control law requires the closed-loop system to achieve at least $6 \mathrm{~dB}$ of gain margin and $45^{\circ}$ of phase margin. The F/A-18 aircraft closed-loop plants under consideration are multivariable; hence, both disk margin and multivariable margin analyses are also performed in addition to the classical loop-at-a-time margin analysis.

\section{Classical Gain, Phase, and Delay Margin Analysis}

Classical gain, phase, and delay margins provide robustness margins for each individual feedback channel with all the other loops closed. This loop-at-a-time margin analysis provides insight on the sensitivity of each channel individually. Table 8 provides the classical margins for both the baseline and the revised flight control laws. The results, presented in Table 8 , are based on the uncoordinated $\left(\beta=10^{\circ}\right)$ bank turn maneuver at $\phi=35^{\circ}$ (plant 8 ). This plant results in the worst margins among all the other plants mentioned in Table 4 . The baseline and revised flight control laws

Table 7 Eigenvalue of the F/A-18 linear plant

\begin{tabular}{lcccc}
\hline \hline & \multicolumn{2}{c}{ Nine-state } & \multicolumn{2}{c}{ Six-state } \\
\cline { 2 - 5 } Mode & Eigenvalue & Period/time constant, s & Eigenvalue & Period/time constant, s \\
\hline Short period & $-0.195 \pm 1.66$ & 3.79 & $-0.194 \pm 1.66$ & 3.79 \\
Phugoid & $-0.0509 \pm 0.125$ & 50.3 & $-0.203 \pm 0.933$ & -6.73 \\
Dutch roll & $-0.202 \pm 0.918$ & 6.85 & -0.302 & 3.31 \\
Roll subsidence & -0.307 & 3.25 & -0.0515 & 19.4 \\
Spiral & -0.0209 & 47.8 & - & - \\
Heading angle & 0 & 0 & & \\
\hline \hline
\end{tabular}



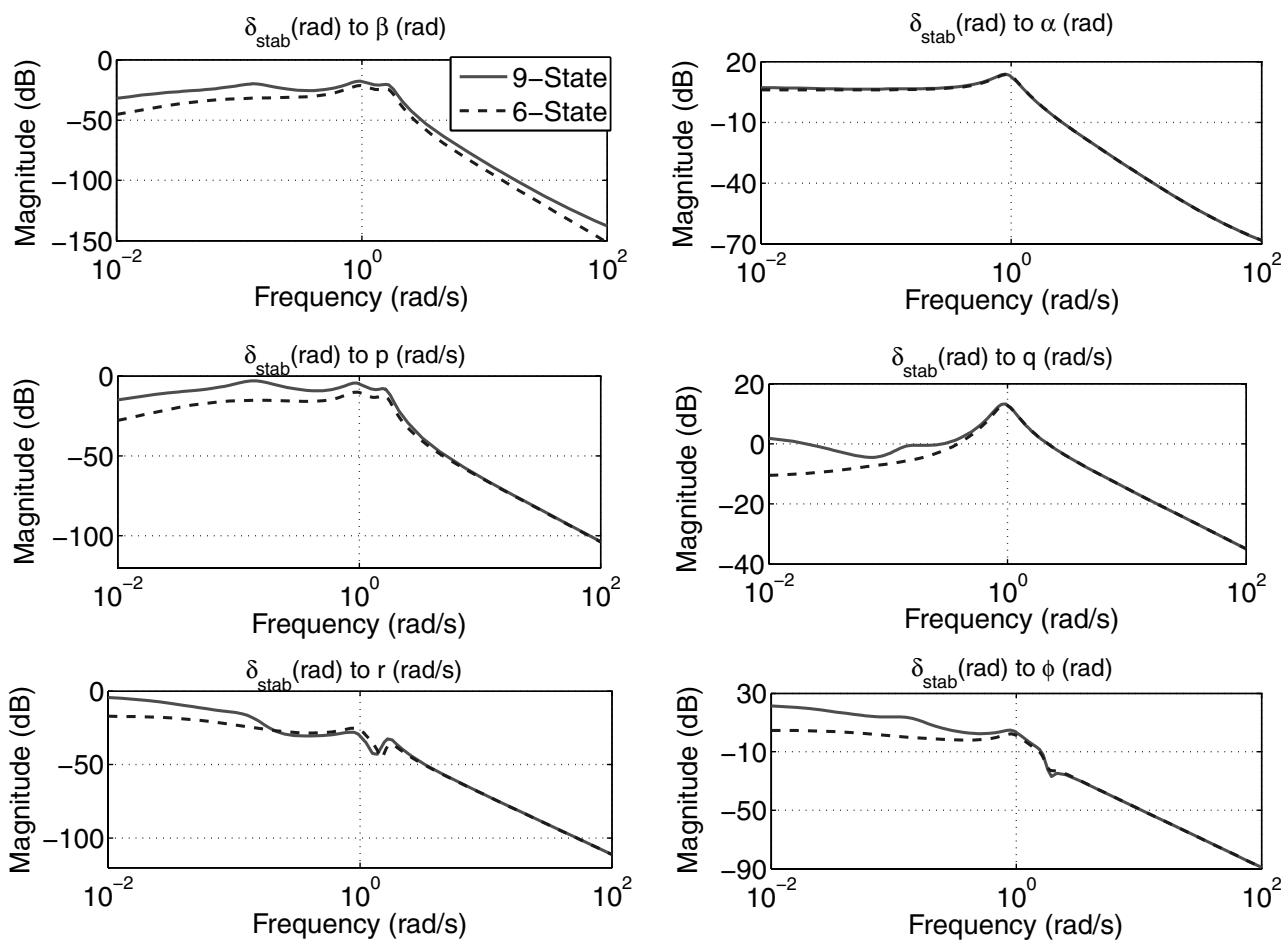

Fig. 7 Bode plot: magnitude comparison between the nine-state and six-state representation.

have very similar classical margins at the input channel. Both flight control laws are very robust and satisfy the minimum requirement of $6 \mathrm{~dB}$ gain margin and $45^{\circ}$ phase margin.

\section{Disk Margin Analysis}

Disk margin analysis provides an estimate of the single-loop robustness to combined gain/phase variations [20]. The disk margin metric is very similar to an exclusion region on a Nichols chart. As with the classical margin calculation, coupling effects between channels may not be captured by this analysis. Table $\underline{9}$ provides the disk gain and phase variations at each loop for both the control laws. The results are based on the uncoordinated bank turn maneuver at $\phi=35^{\circ}$ (plant 8). Again, both the flight control laws achieve similar robustness margin which exceed the desired requirements. The disk margins of the two flight control laws are nearly identical.

\section{Multivariable Disk Margin Analysis}

The multivariable disk margin indicates the robustness of the closed-loop system to simultaneous (across all channels), independent gain and phase variations. This analysis is conservative
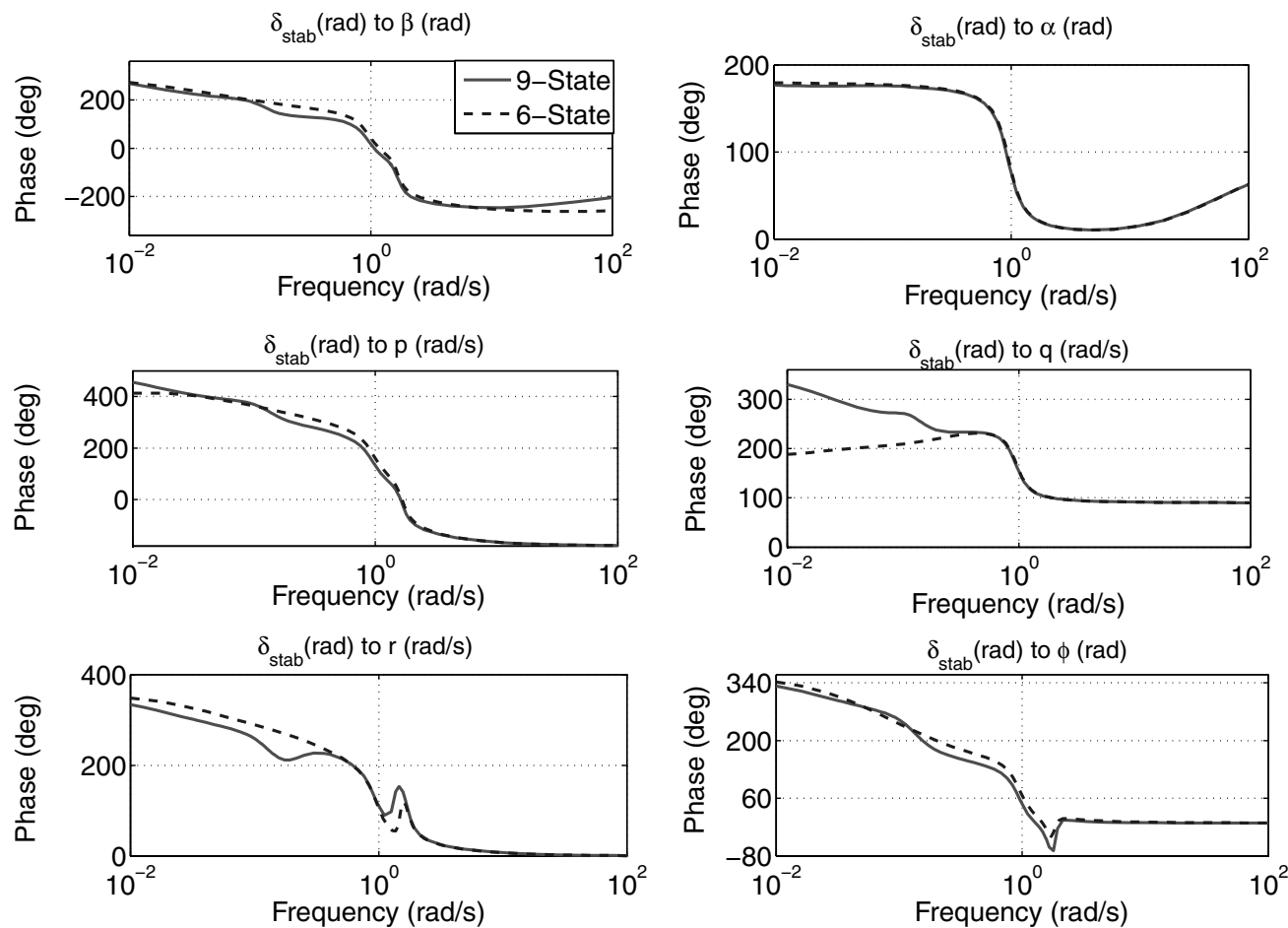

Fig. 8 Bode plot: phase comparison between the nine-state and six-state representation. 
Table 8 Classical gain and phase margin analysis for plant 8

\begin{tabular}{lcc}
\hline \hline Input channel & Baseline & Revised \\
\hline & Aileron & \\
Gain margin & $43.4 \mathrm{~dB}$ & $38.3 \mathrm{~dB}$ \\
Phase margin & $\infty$ & $88.8^{\circ}$ \\
Delay margin & $\infty$ & $0.378 \mathrm{~s}$ \\
& Rudder & \\
Gain margin & $20.8 \mathrm{~dB}$ & $20.5 \mathrm{~dB}$ \\
Phase margin & $68.7^{\circ}$ & $69.5^{\circ}$ \\
Delay margin & $1.97 \mathrm{~s}$ & $1.36 \mathrm{~s}$ \\
& Stabilator & \\
Gain margin & $\infty$ & $\infty$ \\
Phase margin & $66.8^{\circ}$ & $66.8^{\circ}$ \\
Delay margin & $0.0887 \mathrm{~s}$ & $0.0887 \mathrm{~s}$ \\
\hline \hline
\end{tabular}

Table 9 Disk margin analysis for plant 8

\begin{tabular}{lcc}
\hline \hline Input channel & Baseline & Revised \\
\hline Gain margin & $43.4 \mathrm{~dB}$ & $23.0 \mathrm{~dB}$ \\
Phase margin & $89.2^{\circ}$ & $81.9^{\circ}$ \\
& Rudder & \\
Gain margin & $7.15 \mathrm{~dB}$ & $7.92 \mathrm{~dB}$ \\
Phase margin & $42.6^{\circ}$ & $46.2^{\circ}$ \\
& Stabilator & \\
Gain margin & $11.8 \mathrm{~dB}$ & 11.8 \\
Phase margin & $61.2^{\circ}$ & $61.4^{\circ}$ \\
\hline \hline
\end{tabular}

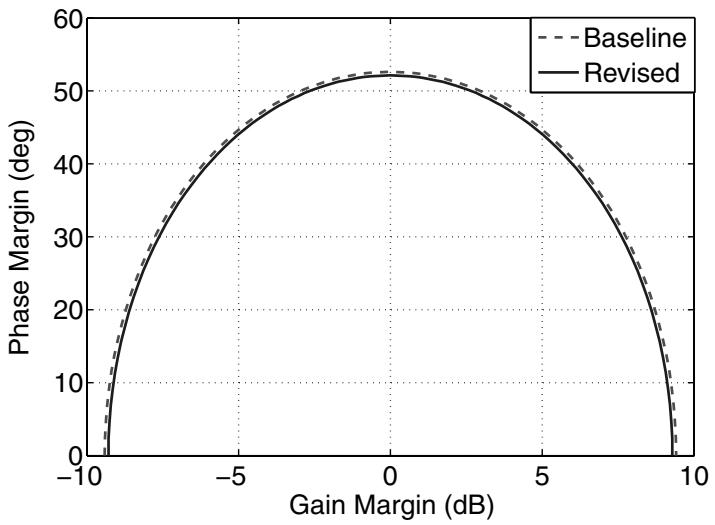

Fig. 9 Multivariable disk margin analysis for coordinated $35^{\circ}$ bank angle turn.

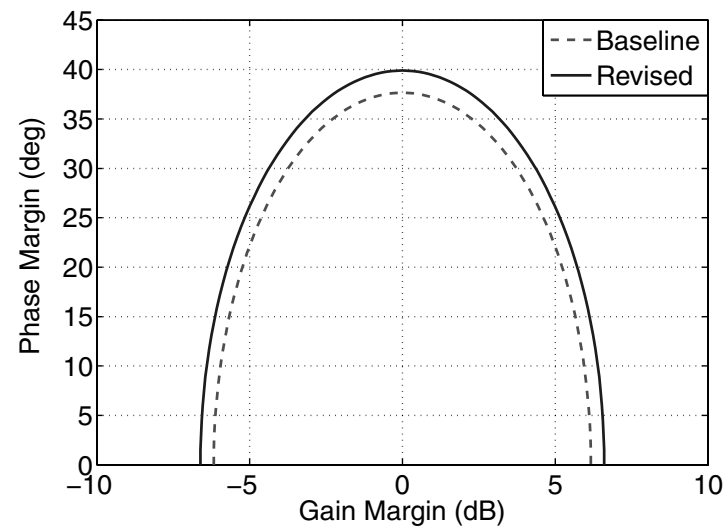

Fig. 10 Multivariable disk margin analysis for uncoordinated $35^{\circ}$ bank angle turn with $10^{\circ}$ sideslip angle. because it allows independent variation of the input channels simultaneously. Figures 9 and 10 present the multivariable disk margin ellipses, respectively, for plant 4 (coordinated turn at $35^{\circ}$ bank angle) and plant 8 (uncoordinated turn at $35^{\circ}$ bank angle). The multivariable disk margin analysis certifies that for simultaneous gain and phase variations in each channel inside the region of the ellipses the closed-loop system remains stable. The multivariable disk margin analysis for steady bank turn maneuvers, Fig. 9, shows both the baseline and the revised flight control laws have similar multivariable margins. In fact, the baseline appears to have slightly better margin than the revised flight control law. For this steady maneuver, both the control laws are robust to gain variation of up to $\approx \pm 9.2 \mathrm{~dB}$ and phase variation of $\approx \pm 52^{\circ}$ across channels.

Figure 10 shows the multivariable disk margin analysis for unsteady bank turn maneuvers. Here, the revised flight control law has a slightly better margin than the baseline flight control law. However, the differences in the margins between the two control laws is not significant enough to conclude which flight control law is susceptible to the falling-leaf motion. Moreover, both the control laws achieve the typical margin requirement specification $(6 \mathrm{~dB}$ gain margin and $45^{\circ}$ phase margin) for the steady maneuver. For the unsteady maneuvers, the gain margin requirement is satisfied (both achieves slightly over $6 \mathrm{~dB})$, but the achieved phase margin $\left(\approx 40^{\circ}\right)$ falls short of the requirement.

\section{Unmodeled Dynamics: Input Multiplicative Uncertainty}

Modeling physical systems accurately in many engineering applications is a challenge. A mathematical model of the physical system usually differs from the actual behavior of the system. The F/A-18 aircraft model presented in this paper is no exception. One approach is to account for the inaccuracies of the modeled aircraft dynamics by unmodeled dynamics entering at the input to the system.

Figure 11 shows the general uncertainty structure of the plant that will be considered in the input multiplicative uncertainty analysis. To assess the performance due to the inaccuracies of the vehicle modeled, multiplicative uncertainty, $W_{I} \Delta_{\mathrm{IM}}$, in all three input channels is introduced. The uncertainty $\Delta_{\text {IM }}$ represents unit norm bounded unmodeled dynamics. The weighting function is set to unity for analysis purpose, $W_{I}=I_{3 \times 3}$. The structured singular value $(\mu)$ is used to analyze the uncertain closed-loop system. The $\frac{1}{\mu}$ value

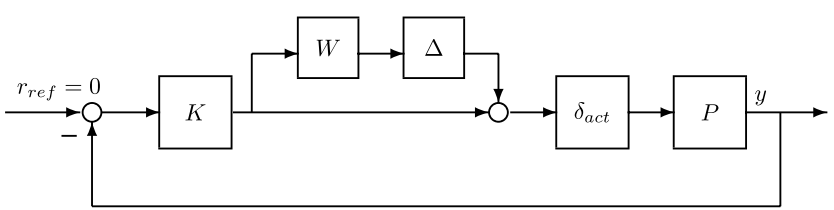

Fig. 11 F/A-18 input multiplicative uncertainty structure.

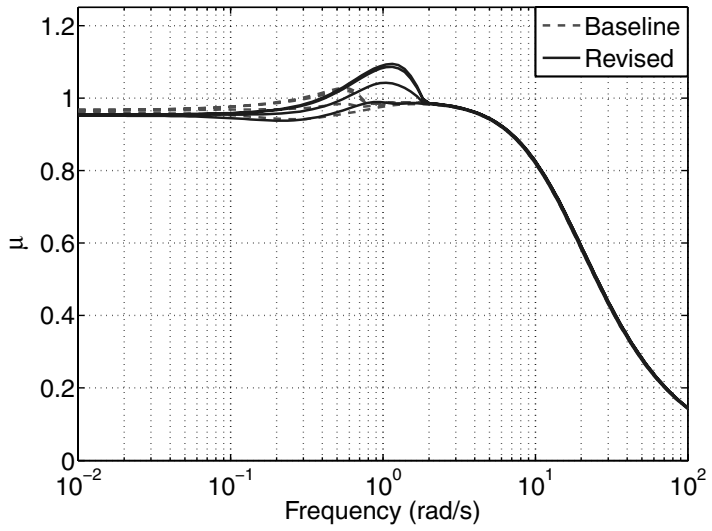

Fig. 12 Diagonal input multiplicative uncertainty: coordinated maneuvers. 
measures the stability margin due to the uncertainty description in the system.

\section{Diagonal Input Multiplicative Uncertainty}

Figures 12 and 13 show the $\mu$ plot of the baseline and revised closed-loop system for coordinated (plants 1-4) and uncoordinated (plants 5-8) bank maneuvers for the interconnection structure shown in Fig. 11. The uncertainty, $\Delta_{\mathrm{IM}}$, is assumed to have a diagonal structure indicating the presence of uncertainty in each actuation channel but no cross-coupling among the channels. The value of $\mu$ at each frequency $\omega$ is inversely related to the smallest uncertainty which causes the feedback system to have poles at $\pm j \omega$. Thus the largest value on the $\mu$ plot is equal to $1 / k_{m}$ where $k_{m}$ denotes the stability margin. In Fig. 12 , the peak value of $\mu$ is 1.150 $\left(k_{m}=0.8695\right)$ for the revised controller during steady maneuvers. The baseline achieves a peak value of $\mu$ is $1.030\left(k_{m}=0.9708\right)$. The baseline flight control law achieves a slightly better robustness for the coordinated bank turn maneuvers compared with the revised flight control law. Figure 13 shows the peak value of $\mu$ for both the control laws at uncoordinated bank turn maneuvers. Here, the baseline flight controller exhibits a peak $\mu$ value of $1.894\left(k_{m}=0.5279\right)$ and the revised flight controller achieves a $\mu$ value of $1.816\left(k_{m}=0.5506\right)$.

Both the flight control laws exhibit similar robustness or stability margins under diagonal input multiplicative uncertainty for both the coordinated and uncoordinated maneuvers. Overall, the stability margins of both the control laws are excellent and nearly identical.

\section{Full-Block Input Multiplicative Uncertainty}

The input multiplicative uncertainty, $\Delta_{\mathrm{IM}}$, is treated as a full-block uncertainty in the analysis. This uncertainty structure models the effects of dynamic cross-coupling between the channels to determine how well the flight control laws are able to handle the coupling at the

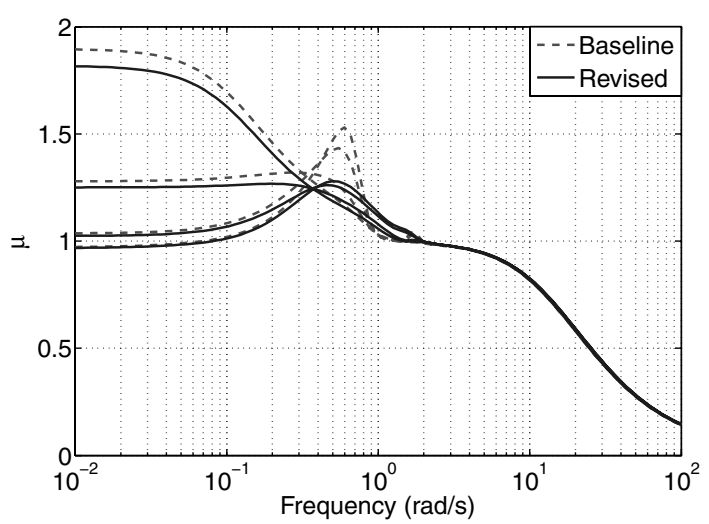

Fig. 13 Diagonal input multiplicative uncertainty: uncoordinated maneuvers.

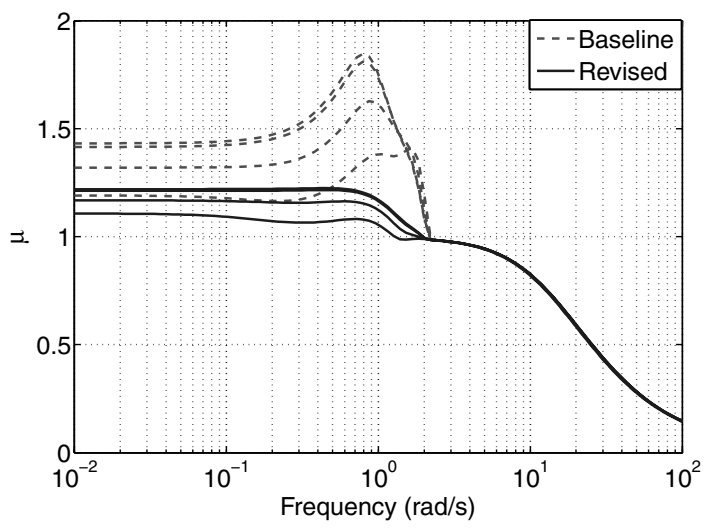

Fig. 14 Full-block input multiplicative uncertainty: coordinated maneuvers. input to the F/A-18 actuators. As mentioned before, the falling-leaf motion is an exaggerated form of in-phase Dutch-roll motion with large coupling in the roll-yaw direction. Increased robustness of the flight control law with respect to the full $\Delta_{\mathrm{IM}}$ is associated with its ability to mitigate the onset of the falling-leaf motion. Figure 14 presents robustness results for coordinated maneuvers (plants $1-\overline{4}$ ), and Fig. 15 presents results for uncoordinated (plants 5-8) maneuvers.

Figure 14 shows the $\mu$ analysis for coordinated maneuvers. In this case, the baseline flight control law achieves a peak $\mu$ value of 1.846 $\left(k_{m}=0.5417\right)$ and the revised flight control law achieves a peak $\mu$ value of $1.220\left(k_{m}=0.8196\right)$. The results indicate the revised flight control law is more robust as compared with the baseline flight control law up to $2 \mathrm{rad} / \mathrm{s}$. Similarly, Fig. 15 shows the $\mu$ analysis for uncoordinated maneuvers. The baseline flight control law achieves a peak $\mu$ value of $3.075\left(k_{m}=0.3252\right)$ and the revised flight control law achieves a peak $\mu$ value of $2.032\left(k_{m}=0.4921\right)$.

Linear robustness analysis with respect to full-block input multiplicative uncertainty across input channels indicate the revised controller is more robust than the baseline design. This implies that the revised controller is better able to handle cross-coupling in the actuation channels. Moreover, both Figs. 14 and 15 show the revised controller provides additional damping to the system around approximately $1 \mathrm{rad} / \mathrm{s}$, while the baseline peaks up around that frequency.

\section{Robustness Analysis to Parametric Uncertainty}

Robustness analysis of flight control system with parametric uncertainty is another important analysis in validating closed-loop robustness and performance [21]. Moreover, robustness assessment of the flight control law due to the variations of aerodynamic coefficients over the flight envelope needs to be considered.

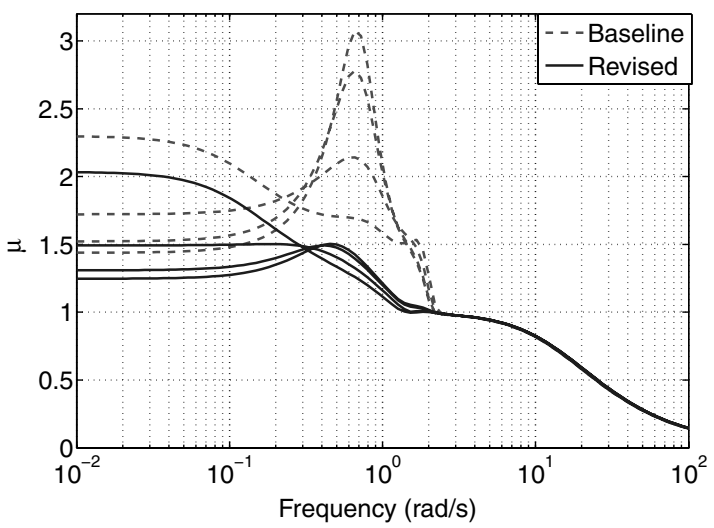

Fig. 15 Full-block input multiplicative uncertainty: uncoordinated maneuvers.

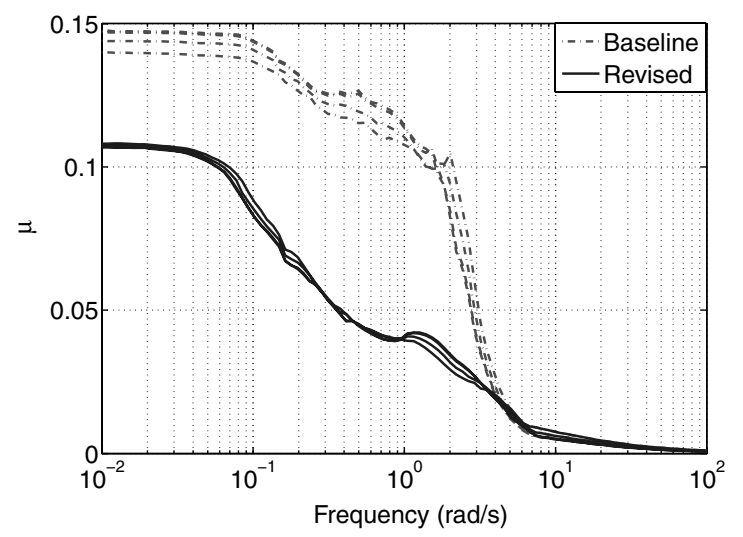

Fig. 16 Real parametric uncertainty in aerodynamic coefficients: coordinated maneuvers. 
Including parametric uncertainty models into the analysis is one approach to address this issue. Both controllers are examined with respect to robustness in the presence of parametric variations in the plant model. To this end, the stability derivatives of the linearized model are represented with $\pm 10 \%$ uncertainty around their nominal values. These perturbed coefficients are chosen carefully to represent the stability characteristics of the F/A-18 aircraft that play an important role in the falling-leaf motion. These terms are related to the entries of the linearized open-loop $A$ matrix. The terms in the lateral directions are: sideforce due to sideslip $\left(Y_{\beta}\right)$; rolling moment due to sideslip $\left(L_{\beta}\right)$; yawing moment due to sideslip $\left(N_{\beta}\right)$; roll damping $\left(L_{p}\right)$; yaw damping $\left(N_{r}\right)$. The following longitudinal terms have also been considered: pitch damping $\left(M_{q}\right)$; normal force due to pitch rate $\left(Z_{q}\right)$; pitch stiffness $\left(M_{\alpha}\right)$. Cook [15] provides a detailed description of these terms. The lateral aerodynamic terms: $Y_{\beta}, L_{\beta}$, $N_{\beta}, L_{p}$, and $N_{r}$ correspond, respectively, to the $(1,1),(3,1),(5,1),(3$, $3)$, and $(5,5)$ entries of the linearized $A$ matrix presented in previous section. The longitudinal aerodynamic terms: $M_{q}, Z_{q}$, and $M_{\alpha}$ correspond, respectively, to the $(4,4),(2,4)$, and $(4,2)$ entries of the same linearized $A$ matrix.

Figures 16 and 17 show the $\mu$ plot of both closed-loop systems with respect to the parametric uncertainty for both coordinated (plants 1-4) and uncoordinated maneuvers (plants 5-8), respectively. In Fig. 16, the stability margin for parametric uncertainty in the aerodynamic coefficients of the revised controller $(\mu=0.1080$ and $\left.k_{m}=9.259\right)$ is approximately 1.3 times larger than that of the baseline controller $\left(\mu=0.1475\right.$ and $\left.k_{m}=6.779\right)$. Figure 17 presents results based on plants $5-8$ for uncoordinated $\left(\beta=10^{\circ}\right)$ maneuvers. In Fig. 17, the stability margin for parametric uncertainty in the aerodynamic coefficients of the revised controller $(\mu=0.2016$ and $\left.k_{m}=4.960\right)$ is approximately 1.3 times larger than that of the baseline controller $\left(\mu=0.2746\right.$ and $\left.k_{m}=3.642\right)$. Hence, the revised flight controller is more robust to uncertainty in aerodynamic derivatives than the baseline design. This is particularly true for the uncoordinated turns. With uncoordinated banking maneuvers, the $\mu$ value for the baseline flight control law peaks up around $0.7 \mathrm{rad} / \mathrm{s}$, while the revised design does not exhibit the peaking behavior. However, both the flight controllers prove to be very robust against the parametric uncertainty in the stability derivatives.

\section{E. Worst-Case Analysis of Flight Control Laws}

The ability of the revised flight control law to damp out the sideslip motion, even during high AOA maneuvers, is key in suppressing the falling-leaf motion [2]. This motivates a comparison between the worst-case performance of the two flight control laws due to disturbances in aileron and rudder channel, uncertainty in the stability derivatives, and their effect on the sideslip. Figure 18 shows the setup of the problem formulation. The one-by-two transfer function of interest is from disturbances in the aileron and rudder channels to sideslip output. $P_{\Delta}$ is the uncertain plant with uncertainty being represented as 1) parametric uncertainty in aerodynamic coefficients and 2) unmodeled dynamics uncertainty.

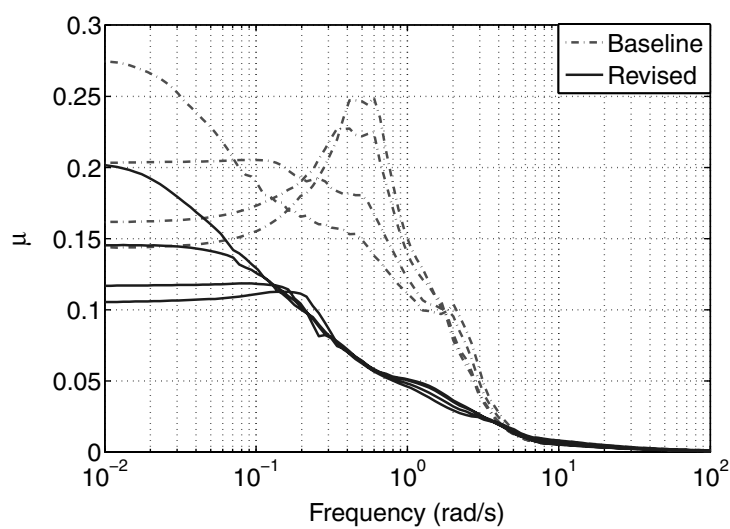

Fig. 17 Real parametric uncertainty in aerodynamic coefficients: uncoordinated maneuvers.

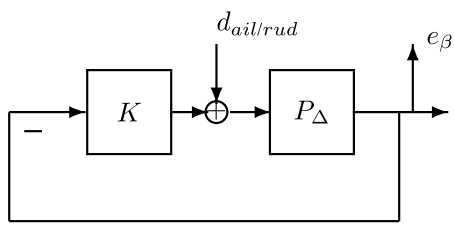

Fig. 18 Setup of worst-case analysis: $d$ indicates the disturbances in rudder and aileron channel and $e_{\beta}$ indicates the sideslip channel.

\section{Worst-Case Parametric Uncertainty Analysis}

The uncertainty, in this case, is associated with stability derivatives of the plant as described in Sec. IV.D. Figure 19 shows a frequencydependent $\mu$ plot of the worst-case gain analysis from the sideslip feedback channel to the aileron and rudder input channel for the coordinated maneuvers. Comparatively, the revised flight control law performs better than the baseline flight control law. The baseline flight control achieves a peak worst-case gain of 1.30 while the revised flight control law achieves a worst-case gain of 0.675 . Figure 20 shows $\mu$ plot of the worst-case gain curve for uncoordinated maneuvers. In this case, the revised flight control law performs substantially better than the baseline design. The worstcase gain of the baseline control law is 2.41 while the revised achieves a value of 0.748 .

In both maneuvers, the revised flight control law damps out the peak in the worst-case gain in sideslip direction while the baseline fails to do so.

\section{Unmodeled Dynamics: Diagonal Input Multiplicative Uncertainty}

The unmodeled dynamics uncertainty is modeled in the actuation channel with no cross-coupling (diagonal input multiplicative uncertainty) between them, as described in Sec. IV.C. The results turn

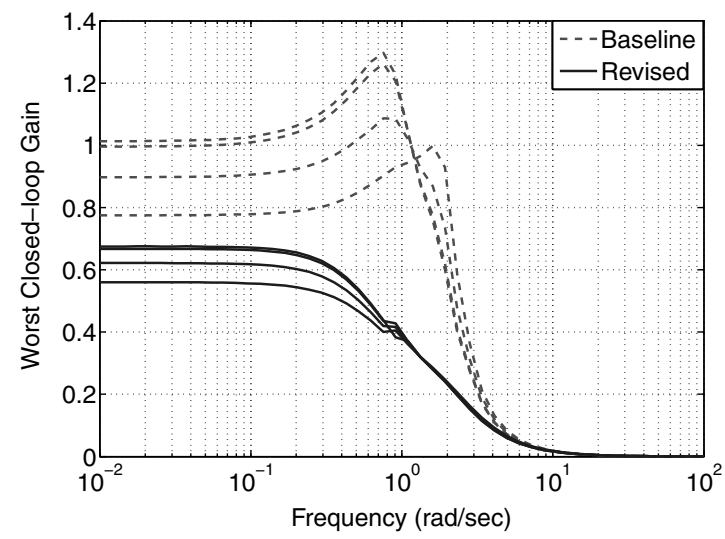

Fig. 19 Worst-case closed-loop gain as a function of frequency under parametric variations: coordinated maneuvers.

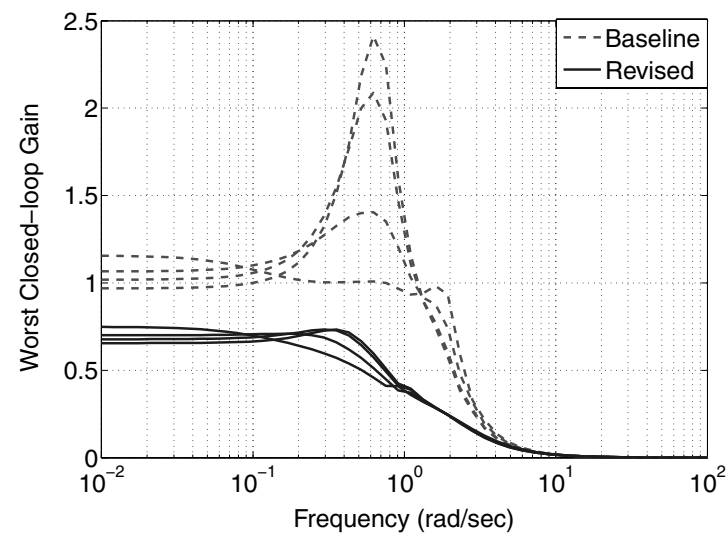

Fig. 20 Worst-case closed-loop gain as a function of frequency under parametric variations: uncoordinated maneuvers. 


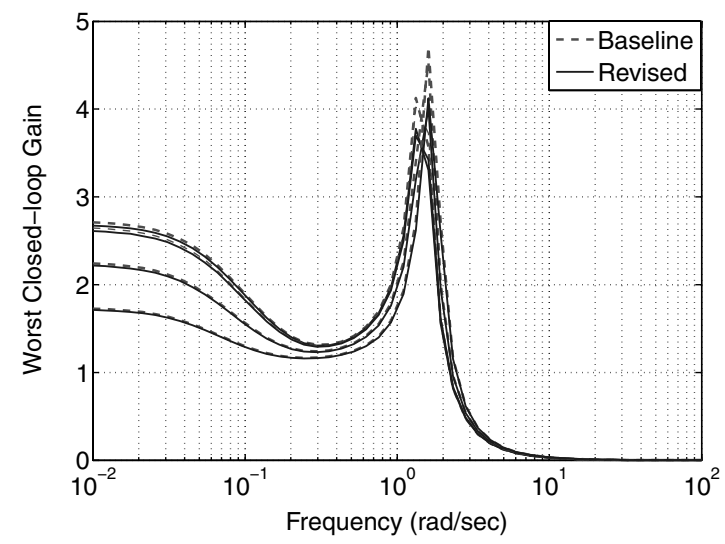

Fig. 21 Worst-case closed-loop gain as a function of frequency under unmodeled dynamics uncertainty: coordinated maneuvers.

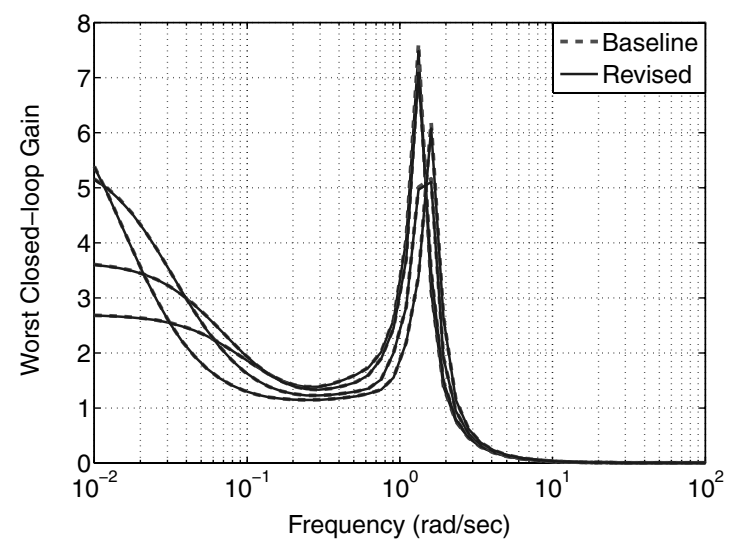

Fig. 22 Worst-case closed-loop gain as a function of frequency under unmodeled dynamics uncertainty: uncoordinated maneuvers.

out to be similar for the full-block input multiplicative uncertainty case. Hence, only diagonal input multiplicative uncertainty results are presented.

In the diagonal input multiplicative uncertainty case for the coordinated maneuvers, the baseline flight control law achieves a slightly higher worst-case gain value compared with the revised flight control law. The revised controller achieves a worst-case gain of 4.12 while the baseline design achieves a gain of 4.70 . Figure 21 shows the frequency-dependent worst-case gain curve of the disturbance rejection properties of the flight control laws. Moreover, Fig. 22 shows the worst-case gain curve for the uncoordinated maneuvers. In this flight condition, the worst-case gain for both the baseline and the revised flight control law is similar. Hence, the results indicated that model error that results in strong cross-coupling will degrade the performance of the baseline controller versus the revised controller.

\section{F. Summary of Linear Analysis Results}

Sections IV.B-IV.E employ various linear analysis techniques to compare the robustness properties of the baseline and the revised flight control laws. The standard loop margin analysis, performed in Section IV.B, shows both the controllers achieve almost identical robustness margin and are very robust. Various $\mu$ analyses are performed in the following subsections with different uncertainty structure in the aircraft plant. Section IV.C analyzes both the controller under the presence of unmodeled dynamics uncertainty in the actuation channel. Both the controllers achiever similar robustness properties under no cross-coupling in the actuation channels. However, the revised design achieves better robustness properties when cross-coupling is modeled in the actuation channels. Similarly, the revised controller is more robust to errors in the aerodynamic coefficients. The worst-case analysis results confirm the $\mu$-analysis results, see Sec. IV.E.

The flight test results have shown that the revised control law is able to damp out the falling-leaf motion while the baseline controller failed to do so. Note that, classical analyses do not indicate any significant difference in the robustness properties between the two control laws. The advance linear analyses did indicate a significant improvement in the robustness properties of the revised control law due to cross-coupling effects at the plant input. This analysis motivates the use of advanced linear robustness analysis tools in the validation and verification process. However, the falling-leaf mode is an inherently nonlinear phenomenon and hence even the advanced linear analysis tools may not be sufficient to accurately assess the closed-loop performance. A follow-on paper [9] uses a nonlinear region of attraction analysis to compare the robustness properties of the baseline and revised control laws.

\section{Nonlinear Simulation Comparison of Control Laws}

Section IV compared the linear robustness properties between the baseline and the revised flight control law in the nonlinear simulation. The linear robustness analyses do not address the issue of how the flight control law will perform under the nonlinearities of the plant. Numerous simulations are performed to compare the two flight control laws. A simulation is performed by perturbing the system from the trimmed flight condition presented in Table 4 for plant 4 , as shown in Figs. 23 and 24 . In this specific simulation, the aileron command from the controller is perturbed by adding a doublet
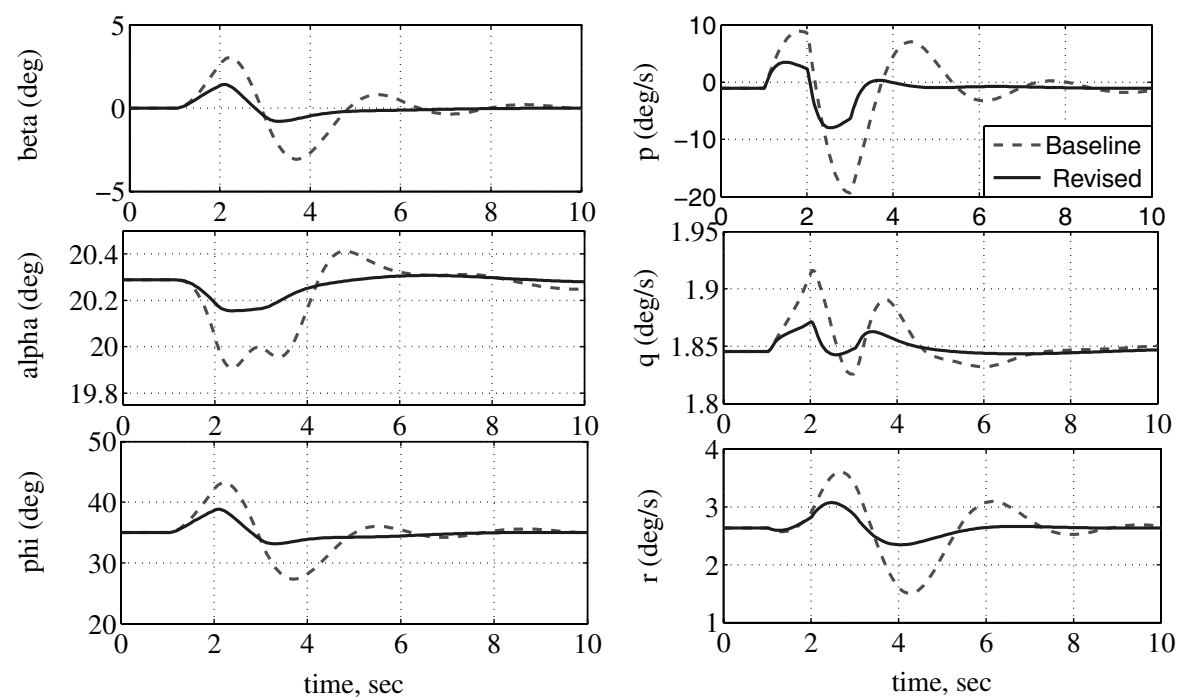

Fig. 23 Nonlinear simulation comparison between baseline and revised flight control law with perturbation in aileron channel. 

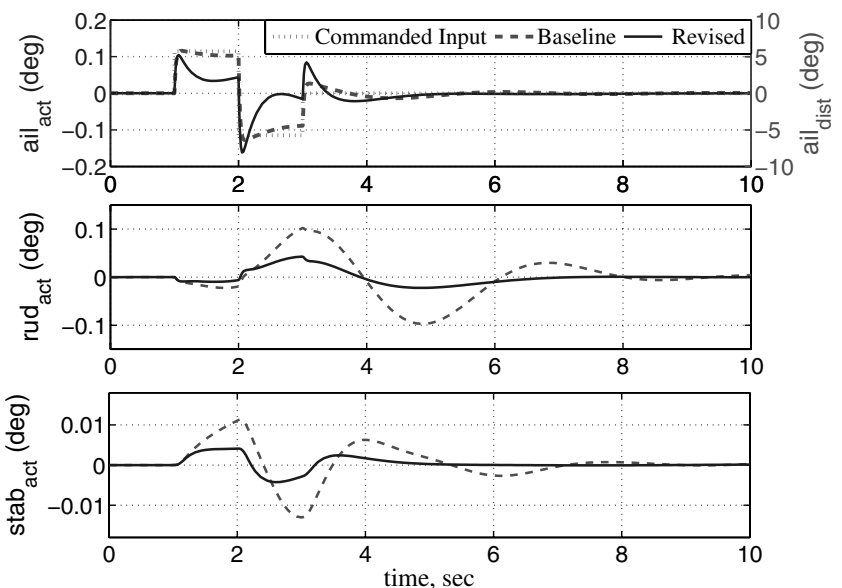

Fig. 24 Actuator dynamics comparison of baseline and revised flight control law with perturbation in aileron channel. Here, ail act $_{\text {indicates }}$ the sum of the command from the controller and the doublet disturbance signal.

disturbance signal of approximately $\pm 5.50^{\circ}$ with a $2 \mathrm{~s}$ duration. Figure 23 shows the state responses of the baseline and the revised flight control law. Notice that the revised flight control law exhibits a more damped response compare to the baseline design. Specifically, the revised control law damps out the sideslip $(\beta)$ oscillation faster than the baseline design. The sideslip damping is key in suppressing the falling-leaf motion [2]. Moreover, the other lateral-directional states, $p, r, \phi$, are also damped out faster and experience smaller deviations compared with the baseline control law. For this particular simulation, the baseline control law also exhibits larger peaks in magnitude response in the longitudinal states, $\alpha, q$. Figure 24 shows the actuator dynamics response during the simulation. Notice that the baseline demands more actuation authority compared with the revised flight control law.

The nonlinear simulation results show that the revised flight control law is better able to damp out the sideslip motion is key to suppressing the falling-leaf motion. On the other hand, the linear analyses have not exhibited any sign of the revised controller being capable of suppressing the falling-leaf motion over the baseline control law. This motivates the necessity to perform nonlinear analysis in validating the stability properties for both the control law design.

\section{Conclusions}

The F/A-18 Hornet aircraft were originally susceptible to the nonlinear falling-leaf mode and this necessitated the design and implementation of a revised flight control law. This paper used linear analysis tools to compare the baseline and revised F/A-18 flight control laws. Standard classical analyses, e.g., gain and phase margins, indicated that the two control laws have similar robustness properties. In contrast, advanced linear analyses, e.g. $\mu$ analysis, indicated that the revised flight control law had significantly better robustness properties. Though both controllers were very robust and did not indicate any susceptibility to departure, the results indicate that the use of advanced multivariable linear analysis tools in the validation and verification process may be beneficial. A follow-on paper analyzes both flight control laws using nonlinear region of attraction estimation.

\section{Appendix: F/A-18 Model and Controllers' Realization}

I. F/A-18 Full Aerodynamic Model

The aerodynamic coefficients presented here have been extracted from several papers [ $\underline{3}-\underline{-}]$. The aerodynamic model of the aircraft is presented here as closed-form expression:
Pitching Moment, $C_{m}=\left(C_{m_{\alpha_{2}}} \alpha^{2}+C_{m_{\alpha_{1}}} \alpha+C_{m_{\alpha_{0}}}\right)$

$$
\begin{aligned}
& +\left(C_{m_{\delta_{\text {stab }_{2}}}} \alpha^{2}+C_{m_{\delta_{\text {stab }_{1}}}} \alpha+C_{m_{\delta_{\text {stab }_{0}}}}\right) \delta_{\text {stab }}+\frac{\bar{c}}{2 V}\left(C_{m_{q_{3}}} \alpha^{3}\right. \\
& \left.+C_{m_{q_{2}}} \alpha^{2}+C_{m_{q_{1}}} \alpha+C_{m_{q_{0}}}\right) q
\end{aligned}
$$

Rolling Moment, $C_{l}=\left(C_{l_{\beta_{4}}} \alpha^{4}+C_{l_{\beta_{3}}} \alpha^{3}+C_{l_{\beta_{2}}} \alpha^{2}+C_{l_{\beta_{1}}} \alpha\right.$

$$
\begin{aligned}
& \left.+C_{l_{\beta_{0}}}\right) \beta+\left(C_{l_{\delta_{\mathrm{ail}_{3}}}} \alpha^{3}+C_{l_{\delta_{\mathrm{ail}_{2}}}} \alpha^{2}+C_{l_{\delta_{\mathrm{ail}_{1}}}} \alpha+C_{l_{\delta_{\mathrm{ail}_{0}}}}\right) \delta_{\text {ail }} \\
& +\left(C_{l_{\delta_{\text {rud }_{3}}}} \alpha^{3}+C_{l_{\delta_{\text {rud }_{2}}}} \alpha^{2}+C_{l_{\delta_{\text {rud }_{1}}}} \alpha+C_{l_{\delta_{\text {rud }_{0}}}}\right) \delta_{\text {rud }} \\
& +\frac{b}{2 V}\left(C_{l_{p_{1}}} \alpha+C_{l_{p_{0}}}\right) p+\frac{b}{2 V}\left(C_{l_{r_{2}}} \alpha^{2}+C_{l_{r_{1}}} \alpha+C_{l_{r_{0}}}\right) r
\end{aligned}
$$

\begin{tabular}{|c|c|c|}
\hline Pitching moment & Rolling moment & Yawing moment \\
\hline $\begin{array}{l}C_{m_{\alpha_{2}}}=-1.2897 \\
C_{m_{\alpha_{1}}}=0.5110 \\
C_{m_{\alpha_{0}}}=-0.0866 \\
C_{m_{\delta_{\text {stab }}}}=0.9338 \\
C_{m_{\delta_{\text {sab }_{1}}}}=-0.3245 \\
C_{m_{\delta_{\text {stab }}}}=-0.9051 \\
C_{m_{q_{3}}}=64.7190 \\
C_{m_{q_{2}}}=-68.5641 \\
C_{m_{q_{1}}}=10.9921 \\
C_{m_{q_{0}}}=-4.1186\end{array}$ & $\begin{array}{l}C_{l_{\beta_{4}}}=-1.6196 \\
C_{l_{\beta_{3}}}=2.3843 \\
C_{l_{\beta_{2}}}=-0.3620 \\
C_{l_{\beta_{1}}}=-0.4153 \\
C_{l_{\beta_{0}}}=-0.0556 \\
C_{l_{\delta_{\text {ail }}}}=0.1989 \\
C_{l_{\delta_{\text {all }_{2}}}}=-0.2646 \\
C_{l_{\delta_{\text {ail }}}}=-0.0516 \\
C_{l_{\delta_{\text {ail }}}}=0.1424 \\
C_{l_{\delta_{\text {rud }}}}=-0.0274 \\
C_{l_{\delta_{\text {rud }}}}=0.0083 \\
C_{l_{\delta_{\text {rud }}}}=0.0014 \\
C_{l_{\delta_{\text {rud }}}}=0.0129 \\
C_{l_{p_{1}}}=0.2377 \\
C_{l_{p_{0}}}=-0.3540 \\
C_{l_{r_{2}}}=-1.0871 \\
C_{l_{r_{1}}}=0.7804 \\
C_{l_{r_{0}}}=0.1983\end{array}$ & 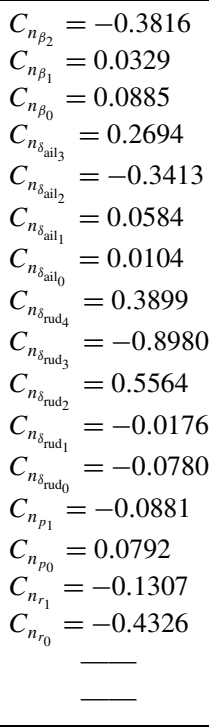 \\
\hline
\end{tabular}

Yawing Moment, $C_{n}=\left(C_{n_{\beta_{2}}} \alpha^{2}+C_{n_{\beta_{1}}} \alpha+C_{n_{\beta_{0}}}\right) \beta$

\begin{tabular}{|c|c|c|}
\hline Sideforce coefficient & Drag force coefficient & Lift force coefficient \\
\hline$C_{Y_{\beta_{2}}}=-0.1926$ & $C_{D_{\alpha_{4}}}=1.4610$ & $C_{L_{\alpha_{3}}}=1.1645$ \\
\hline$C_{Y_{\beta_{1}}}=0.2654$ & $C_{D_{\alpha_{3}}}=-5.7341$ & $C_{L_{\alpha_{2}}}=-5.4246$ \\
\hline$C_{Y_{\beta_{0}}}^{p_{1}}=-0.7344$ & $C_{D_{\alpha_{2}}}=6.3971$ & $C_{L_{\alpha_{1}}}^{u_{2}}=5.6770$ \\
\hline$C_{Y_{\delta_{\mathrm{ail}_{3}}}}=-0.8500$ & $C_{D_{\alpha_{1}}}=-0.1995$ & $C_{L_{\alpha_{0}}}=-0.0204$ \\
\hline$C_{Y_{\delta_{\text {ail }_{2}}}}=1.5317$ & $C_{D_{\alpha_{0}}}=-1.4994$ & $C_{L_{\delta_{\text {sab }_{3}}}=2.1852}$ \\
\hline$C_{Y_{\delta_{\text {dil }}}}=-0.2403$ & $C_{D_{0}}=1.5036$ & $C_{L_{\delta_{\text {stat }}}}=-2.6975$ \\
\hline$C_{Y_{\delta_{\text {ail }_{0}}}}=-0.1656$ & $C_{D_{\delta_{\text {stab }_{3}}}}=-3.8578$ & $C_{L_{\delta_{\text {stab }_{1}}}}=0.4055$ \\
\hline$C_{Y_{\delta_{\text {rud }}}}=0.9351$ & $C_{D_{\delta_{\text {stab }_{2}}}}=4.2360$ & $C_{L_{\delta_{\text {stab }_{0}}}}=0.5725$ \\
\hline$C_{Y_{\delta_{\text {rud }}}}=-1.6921$ & $C_{D_{\delta_{\text {stab }}}}=-0.2739$ & \\
\hline$C_{Y_{\delta_{\text {rud }_{1}}}}=0.4082$ & $C_{D_{\delta_{\text {stab }_{0}}}}=0.0366$ & $\overline{5}$ \\
\hline$C_{Y_{\delta_{\text {rud }_{0}}}}=0.2054$ & & \\
\hline
\end{tabular}

$$
\begin{aligned}
& +\left(C_{n_{\delta_{\mathrm{rud}_{4}}}} \alpha^{4}+C_{n_{\delta_{\mathrm{rud}_{3}}}} \alpha^{3}+C_{n_{\delta_{\mathrm{rud}_{2}}}} \alpha^{2}+C_{n_{\delta_{\mathrm{rud}_{1}}}} \alpha+C_{n_{\delta_{\mathrm{rud}_{0}}}}\right) \delta_{\mathrm{rud}} \\
& +\left(C_{n_{\delta_{\mathrm{ail}_{3}}}} \alpha^{3}+C_{n_{\delta_{\mathrm{ail}_{2}}}} \alpha^{2}+C_{n_{\delta_{\mathrm{ail}_{1}}}} \alpha+C_{n_{\delta_{\mathrm{ail}_{0}}}}\right) \delta_{\mathrm{aill}} \\
& +\frac{b}{2 V}\left(C_{n_{p_{1}}} \alpha+C_{n_{p_{0}}}\right) p+\frac{b}{2 V}\left(C_{n_{r_{1}}} \alpha+C_{n_{r_{0}}}\right) r
\end{aligned}
$$

Table A1 Aerodynamic moment coefficients

Table A2 Aerodynamic force coefficients 
Sideforce Coefficient, $C_{Y}=\left(C_{Y_{\beta_{2}}} \alpha^{2}+C_{Y_{\beta_{2}}} \alpha+C_{Y_{\beta_{0}}}\right) \beta$

$$
\begin{aligned}
& +\left(C_{Y_{\delta_{\text {ail }_{3}}}} \alpha^{3}+C_{Y_{\delta_{\text {ail }_{2}}}} \alpha^{2}+C_{Y_{\delta_{\mathrm{ail}_{1}}}} \alpha+C_{Y_{\delta_{\text {ail }_{0}}}}\right) \delta_{\text {ail }} \\
& +\left(C_{Y_{\delta_{\text {rud }_{3}}}} \alpha^{3}+C_{Y_{\delta_{\text {rud }_{2}}}} \alpha^{2}+C_{Y_{\delta_{\text {rud }_{1}}}} \alpha+C_{Y_{\delta_{\text {rud }_{0}}}}\right) \delta_{\text {rud }}
\end{aligned}
$$

Lift Coefficient, $C_{L}=\left(C_{L_{\alpha_{3}}} \alpha^{3}+C_{L_{\alpha_{2}}} \alpha^{2}+C_{L_{\alpha_{1}}} \alpha\right.$

$$
\begin{aligned}
& \left.+C_{L_{\alpha_{0}}}\right) \cos \left(\frac{2 \beta}{3}\right)+\left(C_{L_{\delta_{\text {stab }_{3}}}} \alpha^{3}+C_{L_{\delta_{\text {stab }_{2}}}} \alpha^{2}+C_{L_{\delta_{\text {stab }_{1}}}} \alpha\right. \\
& \left.+C_{L_{\delta_{\text {stab }_{0}}}}\right) \delta_{\text {stab }}
\end{aligned}
$$

Drag Coefficient, $C_{D}=\left(C_{D_{\alpha_{4}}} \alpha^{4}+C_{D_{\alpha_{3}}} \alpha^{3}+C_{D_{\alpha_{2}}} \alpha^{2}+C_{D_{\alpha_{1}}} \alpha\right.$

$$
\begin{aligned}
& \left.+C_{D_{\alpha_{0}}}\right) \cos \beta+C_{D_{0}}+\left(C_{D_{\delta_{\text {stab }_{3}}}} \alpha^{3}+C_{D_{\delta_{\text {stab }_{2}}}} \alpha^{2}\right.
\end{aligned}
$$

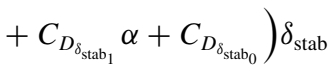

\section{Controller Realization}

The state-space realization of both the baseline and the revised control laws are presented here. The controller $K=\left[\begin{array}{cc}A_{c} & B_{c} \\ C_{c} & D_{C}\end{array}\right]$ where $\dot{x}_{c}=A_{c} x_{c}+B_{c} y$ and $u_{3}=C_{c} x_{c}+D_{c} y$ describes the controllers' state-space realization with $u_{3}$ and $y$ as described in Eq. (10) in Sec. IV.A.

\section{A. Baseline Controller Realization}

The state-space realization of the baseline controller is presented. The baseline flight control law does not require $\beta$ and $\dot{\beta}$ feedback:

$$
\left[\begin{array}{ll}
A_{c} & B_{c} \\
C_{c} & D_{c}
\end{array}\right]=\left[\begin{array}{cccccccc}
-1 & 0 & 0 & 4.9 & 0 & 0 & 0 & 0 \\
0 & 0 & 0.8 & 0 & 0 & 0 & 0 & 0 \\
-1 & -0.5 & 0 & -1.1 & 0 & 0 & 0 & 0 \\
0 & 0 & 0 & 0 & -0.8 & 0 & -8 & 0
\end{array}\right]
$$

\section{B. Revised Controller Realization}

The state-space realization of the revised controller is presented:

$$
\left[\begin{array}{ll}
A_{c} & B_{c} \\
C_{c} & D_{c}
\end{array}\right]=\left[\begin{array}{cccccccc}
-1 & 0 & 0 & 4.9 & 0 & 0 & 0 & 0 \\
0 & 0 & 0.8 & 0 & 0 & 2 & 0 & 0.5 \\
-1 & -0.5 & 0 & -1.1 & 0 & 0 & 0 & 0 \\
0 & 0 & 0 & 0 & -0.8 & 0 & -8 & 0
\end{array}\right]
$$

\section{Linear Plant}

The linear plant described in Eq. (8) is provided next. This linear plant is around the trim points mentioned in Table 4 for plant 4 and plant 8. Refer to Eq. (3) for the ordering of the states and inputs.

A. Coordinated $35^{\circ}$ Bank Turn: Plant 4

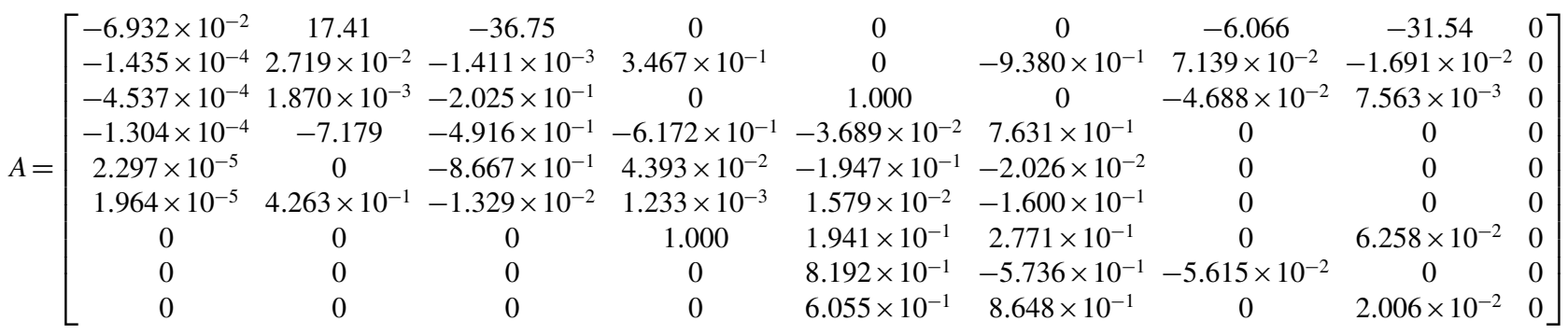

$$
\begin{aligned}
& B=\left[\begin{array}{cccc}
0 & 0 & -7.560 & 9.067 \times 10^{-4} \\
-6.952 \times 10^{-3} & 1.293 \times 10^{-2} & 0 & 0 \\
0 & 0 & -3.425 \times 10^{-2} & -9.577 \times 10^{-7} \\
4.249 & 5.989 \times 10^{-1} & 0 & 0 \\
0 & 0 & -1.796 & 0 \\
-7.287 \times 10^{-2} & -2.877 \times 10^{-1} & 0 & 0 \\
0 & 0 & 0 & 0 \\
0 & 0 & 0 & 0 \\
0 & 0 & 0 & 0
\end{array}\right]
\end{aligned}
$$

$$
C=\left[\begin{array}{ccccccccc}
0 & -5.758 \times 10^{-1} & 0 & 0 & 0 & 0 & 0 & 0 & 0 \\
0 & 0 & 0 & 1 & 0 & 0 & 0 & 0 & 0 \\
0 & 0 & 0 & 0 & 0 & 1 & 0 & 0 & 0 \\
0 & 0 & 1 & 0 & 0 & 0 & 0 & 0 & 0 \\
0 & 1 & 0 & 0 & 0 & 0 & 0 & 0 & 0 \\
0 & 0 & 0 & 0 & 1 & 0 & 0 & 0 & 0 \\
0 & 2.719 \times 10^{-2} & -1.411 \times 10^{-3} & 3.467 \times 10^{-1} & 0 & -9.380 \times 10^{-1} & 7.139 \times 10^{-2} & 0 & 0
\end{array}\right]
$$

$$
D=\left[\begin{array}{cccc}
-1.298 \times 10^{-1} & -1.610 \times 10^{-1} & 0 & 0 \\
0 & 0 & 0 & 0 \\
0 & 0 & 0 & 0 \\
0 & 0 & 0 & 0 \\
0 & 0 & 0 & 0 \\
0 & 0 & 0 & 0 \\
-6.952 \times 10^{-3} & 1.293 \times 10^{-2} & 0 & 0
\end{array}\right]
$$




$$
A=\left[\begin{array}{ccccccccc}
-7.921 \times 10^{-2} & 7.516 & -35.84 & 0 & 0 & 0 & -1.914 & -32.12 & 0 \\
-1.635 \times 10^{-4} & 2.847 \times 10^{-2} & -1.650 \times 10^{-2} & 3.649 \times 10^{-1} & 0 & -9.310 \times 10^{-1} & 7.219 \times 10^{-2} & -3.414 \times 10^{-3} & 0 \\
-4.659 \times 10^{-4} & 5.902 \times 10^{-3} & -1.998 \times 10^{-1} & -1.642 \times 10^{-1} & 1.000 & -6.434 \times 10^{-2} & -4.643 \times 10^{-2} & 5.671 \times 10^{-3} & 0 \\
-1.441 \times 10^{-4} & -7.211 & -2.116 & -6.067 \times 10^{-1} & -3.938 \times 10^{-2} & 7.608 \times 10^{-1} & 0 & 0 \\
2.667 \times 10^{-5} & 0 & -9.807 \times 10^{-1} & 4.689 \times 10^{-2} & -2.031 \times 10^{-1} & -2.487 \times 10^{-2} & 0 & 0 \\
2.062 \times 10^{-5} & 3.995 \times 10^{-1} & -2.621 \times 10^{-1} & 1.188 \times 10^{-3} & 1.938 \times 10^{-2} & -1.607 \times 10^{-1} & 0 & 0 & 0 \\
0 & 0 & 0 & 1.000 & 2.254 \times 10^{-1} & 3.219 \times 10^{-1} & 0 & 6.939 \times 10^{-2} & 0 \\
0 & 0 & 0 & 0 & 8.192 \times 10^{-1} & -5.736 \times 10^{-1} & -6.011 \times 10^{-2} & 0 & 0 \\
0 & 0 & 0 & 0 & 6.163 \times 10^{-1} & 8.801 \times 10^{-1} & 0 & 2.537 \times 10^{-2} & 0
\end{array}\right]
$$

$$
\begin{aligned}
& B=\left[\begin{array}{cccc}
-3.768 \times 10^{-1} & 7.476 \times 10^{-1} & -8.062 & 8.863 \times 10^{-4} \\
-6.106 \times 10^{-3} & 1.211 \times 10^{-2} & 4.061 \times 10^{-3} & 0 \\
0 & 0 & -3.338 \times 10^{-2} & -1.023 \times 10^{-6} \\
4.114 & 5.945 \times 10^{-1} & 0 & 0 \\
0 & 0 & -1.782 & 0 \\
-7.977 \times 10^{-2} & -2.758 \times 10^{-1} & 0 & 0 \\
0 & 0 & 0 & 0 \\
0 & 0 & 0 & 0 \\
0 & 0 & 0 & 0
\end{array}\right] \\
& C=\left[\begin{array}{ccccccccc}
0 & -5.758 \times 10^{-1} & 0 & 0 & 0 & 0 & 0 & 0 & 0 \\
0 & 0 & 0 & 1 & 0 & 0 & 0 & 0 & 0 \\
0 & 0 & 0 & 0 & 0 & 1 & 0 & 0 & 0 \\
0 & 0 & 1 & 0 & 0 & 0 & 0 & 0 & 0 \\
0 & 1 & 0 & 0 & 0 & 0 & 0 & 0 & 0 \\
0 & 0 & 0 & 0 & 1 & 0 & 0 & 0 & 0 \\
0 & 2.847 \times 10^{-2} & 1.650 \times 10^{-2} & 3.649 \times 10^{-1} & 0 & -9.310 \times 10^{-1} & 7.219 \times 10^{-2} & 0 & 0
\end{array}\right]
\end{aligned}
$$

$$
D=\left[\begin{array}{cccc}
-1.298 \times 10^{-1} & -1.610 \times 10^{-1} & 0 & 0 \\
0 & 0 & 0 & 0 \\
0 & 0 & 0 & 0 \\
0 & 0 & 0 & 0 \\
0 & 0 & 0 & 0 \\
0 & 0 & 0 & 0 \\
-6.106 \times 10^{-3} & 1.211 \times 10^{-2} & 4.061 \times 10^{-3} & 0
\end{array}\right]
$$

\section{Acknowledgments}

This research was partially supported under the NASA Langley NASA Research Announcement contract NNH077ZEA001N entitled "Analytical Validation Tools for Safety Critical Systems." The technical contract monitor is Christine Belcastro. We would like to thank John V. Foster at NASA Langley for providing insight into the simulation modeling of the F/A-18 aircraft. We would also like to thank Ufuk Topcu at California Institute of Technology and Andrew Packard at University of California at Berkeley for useful discussions. We would also like to thank the reviewers and the editors for their insightful comments, which have strengthened the paper.

\section{References}

[1] Jaramillo, P. T., and Ralston, J. N., "Simulation of the F/A-18D FallingLeaf," AIAA Atmospheric Flight Mechanics Conference, 1996, pp. $756-766$.

[2] Heller, M., David, R., and Holmberg, J., "Falling-Leaf Motion Suppression in the F/A-18 Hornet with Revised Flight Control Software," AIAA Aerospace Sciences Meeting, AIAA Paper 2004-542, 2004
[3] Napolitano, M. R., Paris, A. C., and Seanor, B. A., "Estimation of the Longitudinal Aerodynamic Parameters from Flight Data for the NASA F/A-18 HARV," AIAA Atmospheric Flight Mechanics Conference, AIAA Paper 96-3419-CP, pp. 469-478.

[4] Napolitano, M. R., Paris, A. C., and Seanor, B. A., "Estimation of the Lateral-Directional Aerodynamic Parameters from Flight Data for the NASA F/A-18 HARV," AIAA Atmospheric Flight Mechanics Conference, AIAA Paper 96-3420-CP, 1996, pp. 479-489.

[5] Lluch, C. D., "Analysis of the Out-of-Control Falling-Leaf Motion Using a Rotational Axis Coordinate System,” Master's Thesis, Virginia Polytechnic Inst. and State Univ., Blacksburg, VA, 1998.

[6] Iliff, K. W., and Wang, K.-S. C., "Extraction of Lateral-Directional Stability and Control Derivatives for the Basic F-18 Aircraft at High Angles of Attack," NASA TM-4786, 1997.

[7] Napolitano, M. R., and Spagnuolo, J. M., "Determination of the Stability and Control Derivatives of the F/A-18 HARV from Flight Data Using the Maximum Likelihood Method," NASA CR-193499, 1993.

[8] Carter, B. R., "Time Optimization of High-Performance Combat Maneuvers," Master's Thesis, Naval Postgraduate School, Monterey, CA, 2005

[9] Chakraborty, A., Seiler, P., and Balas, G., "Susceptibility of F/A-18 Flight Controllers to the Falling-Leaf Mode: Nonlinear Analysis," 
Journal of Guidance, Control, and Dynamics, 2010 (submitted for publication).

[10] Balas, G., Packard, A., Seiler, P., and Topcu, U., "Robustness Analysis of Nonlinear Systems," http://www.aem.umn.edu/ AerospaceControl/ [retrieved 10 Oct. 2010].

[11] Buttrill, S. B., Arbuckle, P. D., and Hoffler, K. D., "Simulation Model of a Twin-Tail, High-Performance Airplane," NASA TR TM-107601, 1992.

[12] Wang, K.-S. C., and Illif, K. W., "Retrospective and Recent Examples of Aircraft Parameter Identification at NASA Dryden Flight Research Center," Journal of Aircraft, Vol. 41, No. 4, 2004, pp. 752-764.

[13] Stevens, B., and Lewis, F., Aircraft Control and Simulation, Wiley, New York, 1992, Chap. 2.

[14] Stengel, R., Flight Dynamics, Princeton Univ. Press, Princeton, NJ, 2004, Chap. 3.

[15] Cook, M., Flight Dynamics Principles, Wiley, New York, 1997, Chap. 4.

[16] Iliff, K. W., and Wang, K.-S. C., "Flight-Determined Subsonic Longitudinal Stability and Control Derivatives of the F-18 High Angle of Attack Research Vehicle (HARV) with Thrust Vectoring," NASATP97-206539, 1997.

[17] Heller, M., Niewoehner, R., and Lawson, P. K., "High Angle of Attack Control Law Development and Testing for the F/A-18E/F Super Hornet," AIAA Guidance, Navigation, and Control Conference, AIAA Paper 1999-4051, 1999, pp. 541-551.

[18] Ostroff, A. J., Hoffler, K. D., and Proffitt, M. S., "High-Alpha Research Vehicle (HARV) Longitudinal Controller: Design, Analyses, and Simulation Results," NASA TR 3446.

[19] Davidson, J. B., Murphy, P. C., Lallman, F. J., Hoffler, K. D., and Bacon, B. J., "High-Alpha Research Vehicle Lateral-Directional Control Law Description, Analyses, and Simulation Results," NASA TP-1998208465.

[20] Balas, G., Chiang, R., Packard, A., and Safonov, M., Robust Control Toolbox, MathWorks, Natwick, MA, 2008.

[21] Heller, M., Niewoehner, R., and Lawson, P. K., "On the Validation of Safety Critical Aircraft Systems, Part 1: An Overview of Analytical and Simulation Methods," AIAA Guidance, Navigation, and Control Conference, AIAA Paper 2003-5559, 2003. 